\title{
Non-geometric backgrounds based on topological interfaces
}

\author{
Yuji Satoh $^{a}$ and Yuji Sugawara ${ }^{b}$ \\ ${ }^{a}$ Institute of Physics, University of Tsukuba, \\ Ibaraki 305-8571, Japan \\ ${ }^{b}$ Department of Physical Sciences, College of Science and Engineering, \\ Ritsumeikan University, \\ Shiga 525-857\%, Japan \\ E-mail: ysatoh@het.ph.tsukuba.ac.jp, ysugawa@se.ritsumei.ac.jp
}

ABSTRACT: We study simple models of the world-sheet CFTs describing non-geometric backgrounds based on the topological interfaces, the 'gluing condition' of which imposes T-duality- or analogous twists. To be more specific, we start with the torus partition function on a target space $S^{1}$ [base] $\times\left(S^{1} \times S^{1}\right)$ [fiber] with rather general values of radii. The fiber CFT is defined by inserting the twist operators consisting of the topological interfaces which lie along the cycles of the world-sheet torus according to the winding numbers of the base circle. We construct the partition functions involving such duality twists. The modular invariance is achieved straightforwardly, whereas 'unitarization' is generically necessary to maintain the unitarity. We demonstrate it in the case of the equal fiber radii. The resultant models are closely related to the CFTs with the discrete torsion. The unitarization is also physically interpreted as multiple insertions of the twist/interface operators along various directions.

Keywords: Conformal Field Models in String Theory, Space-Time Symmetries, Superstring Vacua, String Duality

ARXIV EPRINT: 1502.05776 


\section{Contents}

1 Introduction 1

2 Preliminaries 3

2.1 Partition functions of compact bosons 3

2.2 Orbifolding 4

2.3 Topological interfaces 5

$\begin{array}{lll}3 & \text { Construction of the models } & 7\end{array}$

$\begin{array}{ll}3.1 \text { Basic set up } & 7\end{array}$

$3.2 N=M=1$ case $\quad 9$

3.3 General $N, M \quad 11$

$\begin{array}{lll}3.4 & \text { Interface operator } \mathcal{I}^{(-)} \text {along various cycles } & 14\end{array}$

$\begin{array}{ll}3.5 & \text { Relation to the ' } I^{(+)} \text {-twisted' models } \\ \end{array}$

4 Unitarized models $\quad 16$

$\begin{array}{lll}4.1 & \text { Definition of the unitarized model } & 17\end{array}$

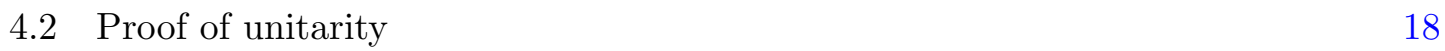

4.3 Interpretation of the unitarized model: multiple insertions of the interface $\begin{array}{ll}\text { operators } & 23\end{array}$

5 Summary and discussion $\quad 24$

$\begin{array}{ll}\text { A Conventions for theta functions and some useful formulas } & 27\end{array}$

$\begin{array}{lr}\text { B Diagonal part operator } & 28\end{array}$

\section{Introduction}

String theory admits the backgrounds which are non-geometric. They are rather ubiquitous, and constitute key ingredients in understanding intrinsic nature of quantum strings, such as string vacua and dualities. Of particular interest among them are the backgrounds whose transition functions involve duality transformations [1-3]. Since the dualities relate different backgrounds, the notion of the Riemannian geometry is generally lost. In the case of T-duality, they thus result in 'T-folds' [4]. One may also consider the backgrounds with the fluxes which are not obtained by a geometric compactification from a higher dimensional theory, namely, non-geometric fluxes. These are often related to the geometric ones by dualities, but are not in general [5-7].

Beyond the classical level, the non-geometric backgrounds should be described by the world-sheet conformal field theory (CFT). The asymmetric orbifold CFTs [8] provide an 
important class, where the left- and right-movers of the string feel different geometries. The non-geometric backgrounds involving the duality twist generally lie at the fixed points of moduli, and the CFT description there is expected to be given by certain asymmetric orbifolds [1]. Along this line, the T-folds with or without non-geometric fluxes have been studied by means of the world-sheet approaches, e.g., in [9-13].

In this paper, we take a step forward to study the non-geometric backgrounds and string vacua from the point of view of the exact world-sheet CFT. In particular, we discuss them based on the world-sheet conformal interfaces [14-16]. The conformal interfaces are defined as the one-dimensional defects which preserve the world-sheet conformal symmetry. When one side of the interface is empty, it becomes a conformal boundary. In this sense, the conformal interfaces are regarded as a generalization of the conformal boundaries, which describe the D-branes in string theory. In addition, when the left and right energy momentum tensors are separately continuous across the interfaces, they are called topological [15, 17], since they can be deformed freely on the world-sheet.

The conformal interfaces possess interesting properties. For example, the topological interfaces implement the symmetries and dualities of the CFT, including T-duality $[18,19]$. They thus glue the CFTs which are related to each other by such symmetries or dualities. From the target-space point of view, they may be interpreted as submanifolds in a doubled target-space (bi-brane) [20]. They also induce transformations of D-branes or boundary renormalization group $(\mathrm{RG})$ flows $[17,21]$. Bulk $\mathrm{RG}$ flows can be described by the nontopological interfaces $[22,23]$. The fusion of the conformal interfaces is expected to give a solution-generating algebra in string theory, similarly to the Ehlers-Geroch transformation in general relativity [24].

Since the conformal invariance is a guiding principle of the world-sheet description of string theory, the conformal interface should also be a fundamental object, though its role in string theory is yet to be uncovered. As an attempt in this direction, we shall investigate the modular invariants involving the conformal interfaces. Our discussion below is based on an observation that the topological interfaces induce the twists associated with the symmetries and dualities, which may yield an exact CFT description of T-folds and analogous nongeometric backgrounds. We shall see that this is indeed the case by explicitly constructing a novel type of the modular invariants describing such non-geometric CFT models. For the discussions on the world-sheet conformal interfaces in the context of string theory, see for instance [16, 24-27].

More specifically, we shall focus on the CFT models defined on the background,

$$
S^{1}[\text { base }] \times\left(S^{1} \times S^{1}\right)[\text { fiber }],
$$

in which the topological interfaces act on the 'fiber CFT' when the world-sheet torus wraps around the base circle. In other words, we begin with the background,

$$
\mathbb{R}[\text { base }] \times\left(S^{1} \times S^{1}\right)[\text { fiber }],
$$

and perform the 'twisted compactification' implemented by the operator given by

$$
\mathcal{T}_{2 \pi R_{\mathrm{b}}} \otimes \mathcal{I}_{\text {fiber }}
$$


Here, $\mathcal{T}_{2 \pi R_{\mathrm{b}}}$ denotes the translation along the base-direction, $\mathcal{T}_{2 \pi R_{\mathrm{b}}}: X \mapsto X+2 \pi R_{\mathrm{b}}$, whereas $\mathcal{I}_{\text {fiber }}$ expresses a certain 'twist' operator acting on the fiber sector, which is made up of the topological interfaces. Such a twist combined with the shift in the base of course follows the spirit of the Scherk-Schwarz compactification [28, 29]. However, the operator $\mathcal{I}_{\text {fiber }}$ here does not necessarily correspond to a symmetry of the original CFT. This means that the conformal systems we propose do not always reduce to ordinary orbifold CFTs. In this sense, our present study would shed light on a possibility to construct a novel type of string vacua, and would illustrate a role of the world-sheet conformal interfaces in string theory.

This paper is organized as follows:

after providing the necessary notation and making a brief review on the topological interfaces in section 2, we shall start our construction of the conformal models and partition functions describing non-geometric backgrounds in section 3. We define the relevant models by using the twisted compactification mentioned above. Even though the construction would be natural and the modular invariance is achieved, an issue of unitarity remains except for the simplest case of the T-fold.

Therefore, in section 4, we discuss a 'unitarization' of the models of our interest. The proposed models are closely related to the orbifold CFTs with the discrete torsion [30, 31], and we explicitly demonstrate that they are indeed unitary for the equal fiber-radii. We further discuss how our unitarization is physically interpreted as the multiple insertions of the twist operators consisting of the topological interfaces.

In section 5, we present a summary and discussion.

\section{Preliminaries}

Before presenting our main analysis, we first set up the necessary notation. Through this paper we shall use the $\alpha^{\prime}=1$ convention. We set $\Lambda \equiv \mathbb{Z} \tau+\mathbb{Z}$, where $\tau \in \mathbb{H}$ (upper half plane) is the modulus of the world-sheet torus parametrized as $\tau=\tau_{1}+i \tau_{2}\left(\tau_{1} \in \mathbb{R}, \tau_{2}>0\right)$.

\subsection{Partition functions of compact bosons}

The partition function of a free boson compactified on the circle with radius $R$ should be

$$
\begin{aligned}
Z_{R}(\tau) & =\sum_{\nu \in \Lambda} Z_{R}(\tau \mid \nu), \\
Z_{R}(\tau \mid \nu) & :=\frac{R}{\sqrt{\tau_{2}}|\eta(\tau)|^{2}} e^{-\frac{\pi R^{2}}{\tau_{2}}|\nu|^{2}},
\end{aligned}
$$

where $Z_{R}(\tau \mid \nu)$ represents a contribution from the winding sector specified by $\nu$. Its modular property is expressed as

$$
Z_{R}(\tau+1 \mid \nu)=Z_{R}(\tau \mid \nu), \quad Z_{R}\left(-\frac{1}{\tau} \mid \frac{\nu}{\tau}\right)=Z_{R}(\tau \mid \nu) .
$$


When the radius $R$ can be written as $R=\sqrt{k},\left(k \in \mathbb{Z}_{>0}\right)$, the partition function $Z_{R}(\tau)$ is rewritten in terms of theta functions, ${ }^{1}$

$$
Z_{R}(\tau)=\frac{1}{|\eta(\tau)|^{2}} \sum_{m \in \mathbb{Z}_{2 k}}\left|\Theta_{m, k}(\tau)\right|^{2}
$$

More generally, if $R=\sqrt{\frac{L}{K}},\left(K, L \in \mathbb{Z}_{>0}\right)$ holds (the rational CFT cases), we obtain

$$
Z_{R}(\tau)=\sum_{\substack{r \in \mathbb{Z}_{2 L} \\ s \in \mathbb{Z}_{K}}} Z^{(K L)}[K r, L s](\tau)
$$

with the notation,

$$
Z^{(k)}[u, v](\tau):=\frac{1}{|\eta(\tau)|^{2}} \Theta_{u+v, k}(\tau) \overline{\Theta_{-u+v, k}(\tau)}
$$

\subsection{Orbifolding}

We introduce the operators corresponding to the following two types of orbifolding:

(i) $\mathbb{Z}_{N}$-action of translation: First, we define $\tau_{\gamma}^{(N), R}$ as the operator linearly acting on the function (2.2) as

$$
\tau_{\gamma}^{(N), R} \cdot\left[\sum_{i} c_{i} Z_{R}\left(\tau \mid \nu_{i}\right)\right]:=\sum_{i} c_{i} Z_{R}\left(\tau \mid \nu_{i}+\frac{\gamma}{N}\right), \quad\left({ }^{\forall} \in \Lambda\right),
$$

with arbitrary $c_{i}, \nu_{i}$. Acting on $Z_{R}(\tau)$, the operator $\frac{1}{N} \sum_{j \in \mathbb{Z}_{N}} \tau_{j}^{(N), R}$ implements the projection restricting the Kaluza-Klein (KK) momentum to $n \in N \mathbb{Z}$ after the Poisson resummation. By the modular completion, we then have an identity,

$$
Z_{R / N}(\tau)=\frac{1}{N} \sum_{\gamma \in \Lambda / N \Lambda} \tau_{\gamma}^{(N), R} \cdot Z_{R}(\tau)
$$

When $R=\sqrt{\frac{N}{L}}$, one can express $Z_{R}(\tau)$ by theta functions through (2.5), where $\tau_{\gamma}^{(N), R}$ act as

$$
\tau_{L(a \tau+b)}^{(N), R} \cdot Z^{(N L)}[u, v](\tau)=e^{2 \pi i \frac{b}{N} u} Z^{(N L)}[u, v+L a](\tau) .
$$

We set above $\gamma=L(a \tau+b) \in \Lambda / N \Lambda$ with $a, b \in \mathbb{Z}_{N}$ by assuming $N, L$ are coprime. This form of the action turns out to be useful for our later analysis.

(ii) $\mathbb{Z}_{N}$-action of 'dual translation': We define $\widetilde{\tau}_{\gamma}^{(N), R}$ as the operator linearly acting on the function (2.2) as

$$
\widetilde{\tau}_{\gamma}^{(N), R} \cdot\left[\sum_{i} c_{i} Z_{R}\left(\tau \mid \nu_{i}\right)\right]:=\sum_{i} c_{i} Z_{R}\left(\tau \mid \nu_{i}\right) e^{2 \pi i \frac{1}{N}\left\langle\nu_{i}, \gamma\right\rangle}, \quad\left({ }^{\forall} \gamma \in \Lambda\right),
$$

\footnotetext{
${ }^{1}$ Our conventions of theta functions are summarized in appendix A.
} 
where we introduced the symbol,

$$
\langle\nu, \gamma\rangle:=\frac{1}{\tau_{2}} \operatorname{Im}(\nu \bar{\gamma})
$$

This time, the operator $\frac{1}{N} \sum_{j \in \mathbb{Z}_{N}} \widetilde{\tau}_{j}^{(N), R}$ acts on $Z_{R}(\tau)$ as the projection onto the states with the winding $w \in N \mathbb{Z}$. We then have an identity,

$$
Z_{N R}(\tau)=\frac{1}{N} \sum_{\gamma \in \Lambda / N \Lambda} \widetilde{\tau}_{\gamma}^{(N), R} \cdot Z_{R}(\tau) .
$$

When $R=\sqrt{\frac{L}{N}}$ with $L, N$ being coprime, one can express $Z_{R}(\tau)$ by theta functions. There, $\widetilde{\tau}_{\gamma}^{(N), R}\left(\gamma=L(\tilde{a} \tau+\tilde{b}) \in \Lambda / N \Lambda ; \tilde{a}, \tilde{b} \in \mathbb{Z}_{N}\right)$ act as

$$
\widetilde{\tau}_{L(\tilde{a} \tau+\tilde{b})}^{(N), R} \cdot Z^{(N L)}[u, v](\tau)=e^{2 \pi i \frac{\tilde{b}}{N} v} Z^{(N L)}[u+L \tilde{a}, v](\tau),
$$

similarly to $\tau_{\gamma}^{(N), R}$. We also note a schematic equivalence,

$$
\frac{1}{N} \sum_{\gamma \in \Lambda / N \Lambda} \widetilde{\tau}_{\gamma}^{(N), R}=T \circ \frac{1}{N} \sum_{\gamma \in \Lambda / N \Lambda} \tau_{\gamma}^{(N), 1 / R} \circ T,
$$

where $T$ denotes the T-duality transformation, $R \rightarrow \frac{1}{R}$.

We shall later use the abbreviations $\tau_{\gamma}^{(N)} \equiv \tau_{\gamma}^{(N), R}, \widetilde{\tau}_{\gamma}^{(N)} \equiv \widetilde{\tau}_{\gamma}^{(N), R}$, as long as there is no fear of confusion.

\subsection{Topological interfaces}

Consider two CFTs with central charge $c=1$, which are denoted by ' $\mathrm{CFT}_{1}$ ' and ' $\mathrm{CFT}_{2}$ ', and described by free bosons $X_{1}, X_{2}$ compactified on the circle with radius $R_{1}, R_{2}$, respectively. We assume that there exist two positive integers $k_{1}, k_{2}$ such that

$$
\frac{k_{2} R_{1} R_{2}}{k_{1}}=1
$$

We denote the oscillators of the free bosons $X_{i}$ as $\alpha_{n}^{i}, \tilde{\alpha}_{n}^{i}$, and the Fock vacua as $|n, w ;(i)\rangle$, $\langle n, w ;(i)|$ whose left and right momenta are given by

$$
p_{L}^{(i)}=\frac{n}{R_{i}}+w R_{i}, \quad p_{R}^{(i)}=\frac{n}{R_{i}}-w R_{i}, \quad\left({ }^{\forall} n, w \in \mathbb{Z}\right) .
$$

Then, one has the topological interface operator $I_{12 ;\left(k_{1}, k_{2}\right)}^{(-)}$that glues the world-sheet of $\mathrm{CFT}_{2}$ with that of $\mathrm{CFT}_{1}[16,24],{ }^{2}$

$$
\begin{aligned}
I_{12 ;\left(k_{1}, k_{2}\right)}^{(-)} & :=G_{12 ;\left(k_{1}, k_{2}\right)}^{(-)} \prod_{n=1}^{\infty} e^{\frac{1}{n}\left(\alpha_{-n}^{1} \alpha_{n}^{2}-\tilde{\alpha}_{-n}^{1} \tilde{\alpha}_{n}^{2}\right)} \\
G_{12 ;\left(k_{1}, k_{2}\right)}^{(-)} & :=\sqrt{k_{1} k_{2}} \sum_{r, s \in \mathbb{Z}}\left|k_{1} s, k_{2} r ;(1)\right\rangle\left\langle k_{1} r, k_{2} s ;(2)\right| .
\end{aligned}
$$

\footnotetext{
${ }^{2}$ We simply set to zero the 'moduli' parameters of the topological interface operators through this paper. We also set to plus a possible sign which could appear in the oscillator part, since it is absorbed by exchanging the left and right movers. It is understood that the oscillators $\alpha_{n}^{1}, \tilde{\alpha}_{n}^{1}$ act on the left side of $G_{12 ;\left(k_{1}, k_{2}\right)}^{(-)}$, whereas $\alpha_{n}^{2}, \tilde{\alpha}_{n}^{2}$ on the right side.
} 
Note that the zero-mode part $G_{12 ;\left(k_{1}, k_{2}\right)}^{(-)}$in (2.18) imposes the 'gluing conditions',

$$
p_{L}^{(1)}=p_{L}^{(2)}, \quad p_{R}^{(1)}=-p_{R}^{(2)},
$$

whereas those on the oscillator part are

$$
\alpha_{n}^{1} I_{12 ;\left(k_{1}, k_{2}\right)}^{(-)}=I_{12 ;\left(k_{1}, k_{2}\right)}^{(-)} \alpha_{n}^{2}, \quad \tilde{\alpha}_{n}^{1} I_{12 ;\left(k_{1}, k_{2}\right)}^{(-)}=-I_{12 ;\left(k_{1}, k_{2}\right)}^{(-)} \tilde{\alpha}_{n}^{2} .
$$

Namely, $I^{(-)}$glues two theories related by T-duality. We also note that when $k_{1}>1$ or $k_{2}>1$, the image of $I_{12 ;\left(k_{1}, k_{2}\right)}^{(-)}$is strictly smaller than the whole Hilbert space of $\mathrm{CFT}_{1}$. In other words, $I_{21 ;\left(k_{2}, k_{1}\right)}^{(-)} \cdot I_{12 ;\left(k_{1}, k_{2}\right)}^{(-)}$is not equal to the identity of $\mathrm{CFT}_{2}$, but is rather identified with the projection operator whose image is the invariant subspace satisfying (2.19).

There is another type of the topological interface operator $I^{(+)}$gluing two free boson theories. It is given by taking the T-duality of the $\mathrm{CFT}_{1}$ in the above construction,

$$
S_{R_{1}}^{1} \rightarrow S_{\tilde{R}_{1}}^{1}, \quad\left(\tilde{R}_{1} \equiv 1 / R_{1}\right),
$$

where $S_{R}^{1}$ denotes the circle with radius $R$. The condition $(2.15)$ is then replaced with

$$
\frac{k_{2} R_{2}}{k_{1} R_{1}}=1
$$

and its explicit form is given by

$$
\begin{aligned}
I_{12 ;\left(k_{1}, k_{2}\right)}^{(+)} & :=G_{12 ;\left(k_{1}, k_{2}\right)}^{(+)} \prod_{n=1}^{\infty} e^{\frac{1}{n}\left(\alpha_{-n}^{1} \alpha_{n}^{2}+\tilde{\alpha}_{-n}^{1} \tilde{\alpha}_{n}^{2}\right)} \\
G_{12 ;\left(k_{1}, k_{2}\right)}^{(+)} & :=\sqrt{k_{1} k_{2}} \sum_{r, s \in \mathbb{Z}}\left|k_{2} r, k_{1} s ;(1)\right\rangle\left\langle k_{1} r, k_{2} s ;(2)\right| .
\end{aligned}
$$

The gluing conditions for $I^{(+)}$are written as

$$
\begin{aligned}
p_{L}^{(1)} & =p_{L}^{(2)}, & p_{R}^{(1)} & =p_{R}^{(2)}, \\
\alpha_{n}^{1} I_{12 ;\left(k_{1}, k_{2}\right)}^{(+)} & =I_{12 ;\left(k_{1}, k_{2}\right)}^{(+)} \alpha_{n}^{2}, & \tilde{\alpha}_{n}^{1} I_{12 ;\left(k_{1}, k_{2}\right)}^{(+)} & =I_{12 ;\left(k_{1}, k_{2}\right)}^{(+)} \tilde{\alpha}_{n}^{2} .
\end{aligned}
$$

From the above gluing conditions, one readily finds that the left and right energymomentum tensors are separately preserved across the interface operators. Thus, $I_{12 ;\left(k_{1}, k_{2}\right)}^{( \pm)}$ indeed represent topological interfaces $[15,17]$, which can be deformed freely on the worldsheet. These are special cases of more general $\widehat{u}(1)$-preserving conformal interfaces [16, 24]. The general gluing conditions are concisely written by $O(1,1)$ matrices. The superscripts $( \pm)$ stand for the connected components of $O(1,1)$. The topological interfaces which do not preserve the $\widehat{u}(1)$-symmetries have also been discussed in [32].

Generally, there are two important subclasses of the topological interfaces [18, 19]. One is named the group-like defect. This class of the interfaces implements the symmetries of the CFT. The other, which includes the former, is the duality defect. This class implements the order-disorder dualities or orbifold equivalences. In our case, $I_{12 ;\left(k_{1}, k_{2}\right)}^{( \pm)}$with $k_{1} k_{2}=1$ is group-like and generates the T-dual symmetry. All other $I_{12 ;\left(k_{1}, k_{2}\right)}^{( \pm)}$are duality defects $[26,32]$. Accordingly, our topological interfaces result in two different classes of the 'duality' twists. 


\section{Construction of the models}

Now, let us start the construction of the models describing non-geometric backgrounds based on the topological interfaces.

\subsection{Basic set up}

We start with a conformal system consisting of free bosons whose target space is given by

$$
\text { ['base' } \mathbb{R}] \times\left[\text { 'fiber' } S_{R_{1}}^{1} \times S_{R_{2}}^{1}\right],
$$

where it is assumed that

$$
R_{1} R_{2}=\frac{N}{M}, \quad\left(N, M \in \mathbb{Z}_{>0}, \operatorname{gcd}\{N, M\}=1\right) .
$$

Then, the condition (2.15) is satisfied when setting

$$
k_{1}=N, \quad k_{2}=M
$$

We would like to make a 'twisted' compactification of this system implemented by the operator,

$$
\mathcal{T}_{2 \pi R_{\mathrm{b}}} \otimes \mathcal{I}^{(-)} .
$$

Here, $\mathcal{T}_{2 \pi R_{\mathrm{b}}}$ denotes the shift operator acting on the base space as $X \longmapsto X+2 \pi R_{\mathrm{b}}$. The fiber part $\mathcal{I}^{(-)}$is defined in terms of the topological interface operator $I^{(-)}$by

$$
\mathcal{I}^{(-)}:=\mathcal{P}\left[I_{12}^{(-)} \otimes I_{21}^{(-)}\right] .
$$

The permutation operator $\mathcal{P}$ in the above acts as

$$
\mathcal{P}\left[|\alpha\rangle_{1} \otimes|\beta\rangle_{2}\right]=|\beta\rangle_{2} \otimes|\alpha\rangle_{1}
$$

where $|\alpha\rangle_{1},|\beta\rangle_{2}$ express arbitrary states of $\mathrm{CFT}_{1}$ and $\mathrm{CFT}_{2}$, respectively. We note that $\mathcal{I}^{(-)}$is regarded as a 'self-interface' of the fiber CFT on $S_{R_{1}}^{1} \times S_{R_{2}}^{1}$, and thus any product $\left(\mathcal{I}^{(-)}\right)^{m}$ is well-defined on its Hilbert space. More explicitly, the zero-mode part of $\mathcal{I}^{(-)}$is given as

$$
\mathcal{I}_{(0)}^{(-)} \propto \sum_{r, s \in \mathbb{Z}} \sum_{r^{\prime}, s^{\prime} \in \mathbb{Z}}\left[\left|N r^{\prime}, M s^{\prime} ;(1)\right\rangle \otimes|N s, M r ;(2)\rangle\right]\left[\langle N r, M s ;(1)| \otimes\left\langle N s^{\prime}, M r^{\prime} ;(2)\right|\right] .
$$

As mentioned above, the topological interface $I^{(-)}$induces the duality transformations. Especially, $\mathcal{I}_{(0)}^{(-)}$glues the zero-mode parts so as to interchange the KK momentum (winding) of the $S_{R_{1}}^{1}$-theory with winding (KK momentum) of the $S_{R_{2}}^{1}$-theory. In this way, we observe non-geometric nature due to the duality twist by (3.5). ${ }^{3}$

\footnotetext{
${ }^{3}$ It would be possible that the models constructed here are equivalent to the world-sheet CFT for some geometric orbifolds via T-duality transformations. In fact, as discussed later, this is the case for the simplest case with $N=M=1$, similarly to known examples of T-fold backgrounds. However, in generic cases with $N M>1$, it seems hard to reinterpret our models as CFTs realized geometrically.
} 
Now, our ansatz of the partition function twisted by (3.4) is expressed in the form,

$$
\begin{aligned}
Z(\tau) & =\sum_{\lambda \in \Lambda(\equiv \mathbb{Z} \tau+\mathbb{Z})} Z_{\lambda}^{\text {base }}(\tau) Z_{\lambda}^{\text {fiber }}(\tau) \\
& \equiv \sum_{\lambda \in \Lambda} Z_{R_{\mathrm{b}}}(\tau \mid \lambda) Z_{\lambda}^{\text {fiber }}(\tau)
\end{aligned}
$$

Here, the base part is expanded by the contribution from each winding sector specified by $\lambda$, and the fiber part is correlated according to this base winding $\lambda$. Adopting this winding basis, the twist operator (3.4) induces a shift of the winding due to $\mathcal{T}_{2 \pi R_{\mathrm{b}}}$ as well as the duality twist caused by $\mathcal{I}^{(-)}$. Every time the string wraps around the base cycle, the fiber part thus receives an additional duality twist by $\mathcal{I}^{(-)}$. Consequently, the contribution from the fiber sector with the winding $\lambda=m \in \mathbb{Z}$ (temporal winding) is given by

$$
Z_{(0, m)}^{\text {fiber }}(\tau) \equiv Z_{\lambda=m}^{\text {fiber }}(\tau):=\mathcal{N}_{\lambda} \operatorname{Tr}_{\mathcal{H}_{\text {fiber }}}\left[\left(\mathcal{I}^{(-)}\right)^{|m|} q^{L_{0}-\frac{2}{24}} \bar{q}^{\tilde{L}_{0}-\frac{2}{24}}\right]
$$

where $\mathcal{N}_{\lambda}$ is a normalization constant.

The torus partition functions with multiple insertions of the general $\widehat{u}(1)$-preserving conformal interfaces have been evaluated in [33]. The evaluation of (3.9) is much simpler and, once it is obtained explicitly, we can uniquely determine the fiber partition function with general winding $Z_{(w, m)}^{\text {fiber }}(\tau) \equiv Z_{\lambda=w \tau+m}^{\text {fiber }}(\tau)$ so that the total partition function (3.8) becomes modular invariant. This means that $Z_{\lambda}^{\text {fiber }}(\tau)$ should possess the modular properties,

$$
Z_{\lambda}^{\text {fiber }}(\tau+1)=Z_{\lambda}^{\text {fiber }}(\tau), \quad Z_{\lambda / \tau}^{\text {fiber }}\left(-\frac{1}{\tau}\right)=Z_{\lambda}^{\text {fiber }}(\tau) .
$$

Here, we should note that $\lambda$ and $\tau$ are treated as independent variables. One may rephrase (3.10) in terms of the alternative notation $Z_{(w, m)}^{\mathrm{fiber}}(\tau)$ as

$$
Z_{(w, m)}^{\mathrm{fiber}}(\tau+1)=Z_{(w, w+m)}^{\mathrm{fiber}}(\tau), \quad Z_{(w, m)}^{\mathrm{fiber}}\left(-\frac{1}{\tau}\right)=Z_{(m,-w)}^{\mathrm{fiber}}(\tau)
$$

Based on these relations, one can readily generate the general building blocks $Z_{(w, m)}^{\mathrm{fiber}}(\tau)$ from $Z_{(0, m)}^{\text {fiber }}(\tau)$. We shall also assume the 'parity invariance',

$$
Z_{-\lambda}^{\text {fiber }}(\tau)=Z_{\lambda}^{\text {fiber }}(\tau)
$$

which seems physically natural and in accord with the twist operator (3.4). In particular, the normalization constants should satisfy

$$
\mathcal{N}_{w \tau+m}=\mathcal{N}_{w \tau+(w+m)}=\mathcal{N}_{m \tau-w}=\mathcal{N}_{-(w \tau+m)} .
$$

We later choose these constants suitably.

From now on, let us analyze concrete examples. 


\section{2 $N=M=1$ case}

We first focus on the simplest case of $N=M=1$. This case also serves as a guide for the general cases that we discuss later. It turns out that it suffices to set $\mathcal{N}_{\lambda}=1$ in this case. We also set $R_{1}=1 / R_{2}=R_{\mathrm{f}}$. We then evaluate $Z_{(0, m)}^{\mathrm{fiber}}$ in (3.9) for $m \in 2 \mathbb{Z}+1$ and $m \in 2 \mathbb{Z}-\{0\}$, separately.

$m \in 2 \mathbb{Z}+1$ sectors: we first consider the $m=1$ case. For the zero-mode part, we obtain

$$
\begin{aligned}
Z_{(0,1), \text { zero-mode }}^{\text {fiber }}(\tau) & =\sum_{r, s \in \mathbb{Z}} \sum_{r^{\prime}, s^{\prime} \in \mathbb{Z}} \delta_{r, r^{\prime}} \delta_{s, s^{\prime}} q^{\frac{1}{4}\left(\frac{r}{R_{\mathrm{f}}}+R_{\mathrm{f}} s\right)^{2}} \bar{q}^{\frac{1}{4}\left(\frac{r}{R_{\mathrm{f}}}-R_{\mathrm{f}} s\right)^{2}} q^{\frac{1}{4}\left(\frac{r^{\prime}}{R_{\mathrm{f}}}+R_{\mathrm{f}} s^{\prime}\right)^{2}} \bar{q}^{\frac{1}{4}\left(\frac{r^{\prime}}{R_{\mathrm{f}}}-R_{\mathrm{f}} s^{\prime}\right)^{2}} \\
& =\sum_{r, s \in \mathbb{Z}} q^{\frac{1}{4}\left(\frac{2 r}{\sqrt{2} R_{\mathrm{f}}}+\sqrt{2} R_{\mathrm{f}} s\right)^{2}} \bar{q}^{\frac{1}{4}\left(\frac{2 r}{\sqrt{2} R_{\mathrm{f}}}-\sqrt{2} R_{\mathrm{f}} s\right)^{2}} .
\end{aligned}
$$

In this calculation, only the 'diagonal part' of $\mathrm{CFT}_{1} \otimes \mathrm{CFT}_{2}$ with

$$
p_{L}^{(1)}=p_{L}^{(2)}=\frac{r}{R_{\mathrm{f}}}+s R_{\mathrm{f}}, \quad p_{R}^{(1)}=-p_{R}^{(2)}=\frac{r}{R_{\mathrm{f}}}-s R_{\mathrm{f}}
$$

survives in the trace under the insertion of $\mathcal{I}^{(-)}$. This eventually leads to the zero-mode spectrum of a compact boson of radius $\sqrt{2} R_{\mathrm{f}}$, where the KK momenta are restricted to even numbers, that is, $r^{\prime}=2 r \in 2 \mathbb{Z}$.

The oscillator part also only includes the diagonal part of $\mathrm{CFT}_{1} \otimes \mathrm{CFT}_{2}$, namely,

$$
\begin{aligned}
Z_{(0,1), \text { oscillator }}^{\text {fiber }}(\tau) & =q^{\frac{2}{24}} \frac{2}{q^{24}} \prod_{n, \tilde{n}=1} \frac{1}{1-q^{2 n}} \frac{1}{1-\bar{q}^{2 \tilde{n}}} \\
& =\left|\frac{2 \eta(\tau)}{\theta_{2}(\tau)}\right| \frac{1}{|\eta(\tau)|^{2}} .
\end{aligned}
$$

Combining (3.14) and (3.16), we obtain

$$
\begin{aligned}
Z_{(0,1)}^{\text {fiber }}(\tau) & =\left|\frac{2 \eta(\tau)}{\theta_{2}(\tau)}\right| \frac{1}{|\eta(\tau)|^{2}} \sum_{r, s \in \mathbb{Z}} q^{\frac{1}{4}\left(\frac{2 r}{\sqrt{2} R_{\mathrm{f}}}+\sqrt{2} R_{\mathrm{f}} s\right)^{2}} \bar{q}^{\frac{1}{4}\left(\frac{2 r}{\sqrt{2} R_{\mathrm{f}}}-\sqrt{2} R_{\mathrm{f}} s\right)^{2}} \\
& =\left|\frac{2 \eta(\tau)}{\theta_{2}(\tau)}\right| \frac{1}{2} \sum_{\nu \in \mathbb{Z} \tau+\frac{1}{2} \mathbb{Z}} Z_{\sqrt{2} R_{\mathrm{f}}}(\tau \mid \nu) .
\end{aligned}
$$

In the second line we made use of the Poisson resummation. It is easy to confirm that we reach the same result for any $m \in 2 \mathbb{Z}+1$ :

$$
Z_{(0, m)}^{\mathrm{fiber}}(\tau)=\left|\frac{2 \eta(\tau)}{\theta_{2}(\tau)}\right| \frac{1}{2} \sum_{\nu \in \mathbb{Z} \tau+\frac{1}{2} \mathbb{Z}} Z_{\sqrt{2} R_{\mathrm{f}}}(\tau \mid \nu), \quad\left({ }^{\forall} m \in 2 \mathbb{Z}+1\right) .
$$

The interpretation of the odd sectors as the diagonal part becomes important in the later discussions. 
$m \in 2 \mathbb{Z}-\{0\}$ sectors: the even sectors are easy to compute, since we have $\left(\mathcal{I}^{(-)}\right)^{2}=1$ for the $N=M=1$ case. We then obtain

$$
\begin{aligned}
Z_{(0, m)}^{\text {fiber }}(\tau) & =\frac{1}{|\eta(\tau)|^{4}}\left[\sum_{r, s \in \mathbb{Z}} q^{\frac{1}{4}\left(\frac{r}{R_{\mathrm{f}}}+R_{\mathrm{f}} s\right)^{2}} \bar{q}^{\frac{1}{4}\left(\frac{r}{R_{\mathrm{f}}}-R_{\mathrm{f}} s\right)^{2}}\right]^{2} \\
& =\left[\sum_{\nu \in \Lambda} Z_{R_{\mathrm{f}}}(\tau \mid \nu)\right]^{2} \equiv Z_{(0,0)}^{\text {fiber }}(\tau) .
\end{aligned}
$$

general winding sectors: we finally determine all the winding sectors that are compatible with the modular invariance. Let $\lambda \equiv w \tau+m \in \Lambda$ be the winding of the base circle. Then, it turns out that the partition functions in question are given by

- $\lambda \in 2 \Lambda$ :

$$
Z_{\lambda}^{\mathrm{fiber}}(\tau)=\left[\sum_{\nu \in \Lambda} Z_{R_{\mathrm{f}}}(\tau \mid \nu)\right]^{2}
$$

- $\lambda \in 2 \Lambda+1$ :

$$
Z_{\lambda}^{\mathrm{fiber}}(\tau)=\left|\frac{2 \eta(\tau)}{\theta_{2}(\tau)}\right| \frac{1}{2} \sum_{j \in \mathbb{Z}_{2}} \sum_{\nu \in \Lambda+\frac{j}{2}} Z_{\sqrt{2} R_{\mathrm{f}}}(\tau \mid \nu) .
$$

- $\lambda \in 2 \Lambda+\tau$ :

$$
Z_{\lambda}^{\text {fiber }}(\tau)=\left|\frac{2 \eta(\tau)}{\theta_{4}(\tau)}\right| \frac{1}{2} \sum_{j \in \mathbb{Z}_{2}} \sum_{\nu \in \Lambda+\frac{j}{2} \tau} Z_{\sqrt{2} R_{\mathrm{f}}}(\tau \mid \nu) .
$$

- $\lambda \in 2 \Lambda+\tau+1$ :

$$
Z_{\lambda}^{\text {fiber }}(\tau)=\left|\frac{2 \eta(\tau)}{\theta_{3}(\tau)}\right| \frac{1}{2} \sum_{j \in \mathbb{Z}_{2}} \sum_{\nu \in \Lambda+\frac{j}{2}(\tau+1)} Z_{\sqrt{2} R_{\mathrm{f}}}(\tau \mid \nu) .
$$

The total partition function is obtained by substituting these results into (3.8).

Note that the present model would be identified with an example of the T-folds, that is, the non-geometric backgrounds based on the T-duality twists. We emphasize that this model includes a continuous modulus $R_{\mathrm{f}}$, while most examples in the literature, of which torus partition functions are precisely calculable, are well-defined only at special points of the moduli space (say, the self-dual radius of the circle). Generally, the T-folds lie at the fixed points of the moduli space under the T-duality twists [1]. In our setting, this is translated into the fact that the twist operator $\mathcal{I}^{(-)}$, which is composed of the topological interface operators, is a 'self-interface' acting within one Hilbert space. Indeed, the Kähler modulus of the $T^{2}$ compactification is fixed under the twist induced by $\mathcal{I}^{(-)}$, though the complex structure modulus is not. 


\subsection{General $N, M$}

We next consider more general cases of

$$
R_{1} R_{2}=\frac{N}{M}, \quad\left(N, M \in \mathbb{Z}_{>0}\right), \quad k_{1}=N, \quad k_{2}=M .
$$

It seems again the simplest to first consider the sectors with the base winding $(0, m)$. Relevant calculations are similar to those in the $N=M=1$ case, but we have a few differences:

- For the $m \in 2 \mathbb{Z}+1$ sectors, we find the zero-mode spectrum of radius $\sqrt{2} R_{1}$ with the restriction that $(\mathrm{KK}$ momentum) $\in 2 N \mathbb{Z}$ and (winding) $\in M \mathbb{Z}$, which means

$$
p_{L}=\frac{2 N r}{\sqrt{2} R_{1}}+\sqrt{2} M s R_{1}, \quad p_{R}=\frac{2 N r}{\sqrt{2} R_{1}}-\sqrt{2} M s R_{1}, \quad(r, s \in \mathbb{Z}) .
$$

- For the $m \in 2 \mathbb{Z}-\{0\}$ sectors, we obtain the square of the partition function of a compact boson of radius $R_{1}$ with the restriction that (KK momentum) $\in N \mathbb{Z}$ and (winding) $\in M \mathbb{Z}$, which means

$$
p_{L}=\frac{N r}{R_{1}}+M R_{1} s\left(\equiv M R_{2} r+\frac{N s}{R_{2}}\right), \quad p_{R}=\frac{N r}{R_{1}}-M R_{1} s\left(\equiv M R_{2} r-\frac{N s}{R_{2}}\right), \quad(r, s \in \mathbb{Z}) .
$$

Note that

$$
Z_{(0, m)}^{\mathrm{fiber}}(\tau) \neq Z_{(0,0)}^{\mathrm{fiber}}(\tau), \quad\left({ }^{\forall} m \in 2 \mathbb{Z}-\{0\}\right),
$$

contrary to the $N=M=1$ case. This is because $\left(\mathcal{I}^{(-)}\right)^{2}$ acts as a projection operator when $k_{1}>1$ or $k_{2}>1$.

The restrictions of the zero mode spectra given above are suitably achieved by inserting the orbifolding operators $\tau_{j}^{(*)} \equiv \tau_{j}^{(*), R_{i}}, \widetilde{\tau}_{j}^{(*)} \equiv \widetilde{\tau}_{j}^{(*), R_{i}}$ defined in (2.7), (2.10). Namely, we obtain the fiber partition functions for the temporal winding sectors as:

$$
\begin{aligned}
Z_{(0, m)}^{\mathrm{fiber}}(\tau) & =\mathcal{N}_{m}\left|\frac{2 \eta(\tau)}{\theta_{2}(\tau)}\right| \frac{1}{|\eta(\tau)|^{2}} \sum_{r, s \in \mathbb{Z}} q^{\frac{1}{4}\left(\frac{2 N r}{\sqrt{2} R_{1}}+\sqrt{2} M s R_{1}\right)^{2}} \bar{q}^{\frac{1}{4}\left(\frac{2 N r}{\sqrt{2} R_{1}}-\sqrt{2} M s R_{1}\right)^{2}} \\
& =\mathcal{N}_{m}\left|\frac{2 \eta(\tau)}{\theta_{2}(\tau)}\right| \frac{1}{2 N M} \sum_{j \in \mathbb{Z}_{2 N}} \sum_{\tilde{j} \in \mathbb{Z}_{M}} \tau_{j}^{(2 N)} \widetilde{\tau}_{\tilde{j}}^{(M)} \cdot Z_{\sqrt{2} R_{i}}(\tau) \\
Z_{(0, m)}^{\mathrm{fiber}}(\tau) & \left.=\mathcal{N}_{m} \frac{1}{|\eta(\tau)|^{4}}\left[\sum_{r, s \in \mathbb{Z}} q^{\frac{1}{4}\left(\frac{N r}{R_{1}}+M s R_{1}\right)^{2}} \bar{q}^{\frac{1}{4}\left(\frac{N r}{R_{1}}-M s R_{1}\right)^{2}}\right]^{2}+1\right) . \\
& =\mathcal{N}_{m}\left[\frac{1}{N M} \sum_{j \in \mathbb{Z}_{N}} \sum_{\tilde{j} \in \mathbb{Z}_{M}} \tau_{j}^{(N)} \widetilde{\tau}_{\tilde{j}}^{(M)} \cdot Z_{R_{i}}(\tau)\right]^{2}
\end{aligned}
$$


Here, $i=1$ or 2 . Both expressions are the same due to the relation (3.24). This also implies that the result in (3.29) is rewritten in a symmetric form with respect to $R_{1}$ and $R_{2}$. Moreover, we should note that the normalization constants of the interface operators appearing in (2.18) have been absorbed into the redefinition of $\mathcal{N}_{m}$ in these expressions (3.28), (3.29). For the special case of $m=0$, we of course obtain

$$
Z_{(0,0)}^{\text {fiber }}(\tau)=\mathcal{N}_{0} Z_{R_{1}}(\tau) Z_{R_{2}}(\tau)
$$

The total partition function is now determined by the modular invariance. For notational convenience, we introduce,

$$
g_{\lambda}(\tau):= \begin{cases}\left|\frac{2 \eta(\tau)}{\theta_{2}(\tau)}\right|, & (\lambda \equiv 1 \bmod 2 \Lambda), \\ \left|\frac{2 \eta(\tau)}{\theta_{4}(\tau)}\right|, & (\lambda \equiv \tau \bmod 2 \Lambda), \\ \left|\frac{2 \eta(\tau)}{\theta_{3}(\tau)}\right|, & (\lambda \equiv \tau+1 \bmod 2 \Lambda),\end{cases}
$$

for ${ }^{\forall} \lambda \in \Lambda$. We also define the following set $S^{(K)}[\lambda] \subset \Lambda / K \Lambda$ for ${ }^{\forall} \lambda \in \Lambda,{ }^{\forall} K \in \mathbb{Z}_{>0}$ by

$$
S^{(K)}[\lambda]:=\{\nu \in \Lambda ;\langle\lambda, \nu\rangle=0\} / K \Lambda,
$$

where the symbol $\langle$,$\rangle is defined in (2.11).$

With these preparations, we can write down the partition functions of the fiber CFT in general winding sectors as follows:

even sectors: $(\lambda \in 2 \Lambda-\{0\})$ :

$$
Z_{\lambda}^{\text {fiber }}(\tau)=\mathcal{N}_{\lambda}\left[\frac{1}{N M} \sum_{\alpha \in S^{(N)}[\lambda]} \sum_{\tilde{\alpha} \in S^{(M)}[\lambda]} \tau_{\alpha}^{(N)} \widetilde{\tau}_{\tilde{\alpha}}^{(M)} \cdot Z_{R_{i}}(\tau)\right]^{2} .
$$

odd sectors: $(\lambda \in \Lambda-2 \Lambda)$ :

$$
Z_{\lambda}^{\text {fiber }}(\tau)=\mathcal{N}_{\lambda} g_{\lambda}(\tau) \frac{1}{2 N M} \sum_{\alpha \in S^{(2 N)}[\lambda]} \sum_{\tilde{\alpha} \in S^{(M)}[\lambda]} \tau_{\alpha}^{(2 N)} \widetilde{\tau}_{\tilde{\alpha}}^{(M)} \cdot Z_{\sqrt{2} R_{i}}(\tau) .
$$

Here, $i=1$ or 2 . For $\lambda=0$, the fiber partition function is given by (3.30).

Note that

$$
S^{(N)}[\lambda]=\{\nu=0 \tau+j ; j \in \mathbb{Z}\} / N \Lambda \cong \mathbb{Z}_{N},
$$

for the temporal winding sectors $\lambda=m(\neq 0) \in \mathbb{Z}$, and thus (3.33), (3.34) reduce to the previous ones $(3.29),(3.28)$. Due to the $\mathrm{SL}(2 ; \mathbb{Z})$-invariance of $\langle$,$\rangle in (2.11)$, they also possess the expected modularity (3.10) or (3.11). The commutativity of $\tau_{\alpha}^{(2 N)}$ and $\widetilde{\tau}_{\tilde{\alpha}}^{(M)}$ for general $\lambda$ follows from that for $\lambda=m$.

We next determine the normalization factors $\mathcal{N}_{\lambda}$ for ${ }^{\forall} \lambda \in \Lambda$. We shall choose these constants to be the smallest positive numbers such that the $q$-expansion of $Z_{\lambda}^{\text {fiber }}(\tau)$ is written in the form,

$$
Z_{\lambda}^{\mathrm{fiber}}(\tau)=\sum_{\ell, \tilde{\ell}} \sum_{n, \tilde{n} \in \mathbb{Z}} a(\ell, \tilde{\ell}, n, \tilde{n} ; \lambda) q^{\Delta(\ell)+n} \overline{q^{\tilde{\Delta}(\tilde{\ell})+\tilde{n}}}, \quad|a(\ell, \tilde{\ell}, n, \tilde{n} ; \lambda)| \in \mathbb{Z}_{\geq 0}
$$


for every $\lambda$. We note, for instance, the following $q$-expansion of the function $\left({ }^{\forall} \ell \in \mathbb{Z}-\{0\}\right)$,

$$
\frac{1}{N M} \sum_{\alpha \in S^{(N)}[\ell \tau]} \sum_{\tilde{\alpha} \in S^{(M)}[\ell \tau]} \tau_{\alpha}^{(N)} \widetilde{\tau}_{\tilde{\alpha}}^{(M)} \cdot Z_{R_{1}}(\tau) \equiv \frac{1}{N M} \sum_{j \in \mathbb{Z}_{N}} \sum_{\tilde{j} \in \mathbb{Z}_{M}} \tau_{j \tau}^{(N)} \widetilde{\tau}_{\tilde{j} \tau}^{(M)} \cdot Z_{R_{1}}(\tau),
$$

always includes fractional coefficients taking the values in $\frac{1}{N M} \mathbb{Z}$ up to some phase factors. We thus simply set

$$
\mathcal{N}_{\lambda}:= \begin{cases}(N M)^{2} & \lambda \in 2 \Lambda \\ N M & \lambda \in \Lambda-2 \Lambda\end{cases}
$$

It is easy to confirm that, with this choice of $\mathcal{N}_{\lambda}$, the fiber partition function $Z_{\lambda}^{\text {fiber }}(\tau)$ is actually written in the form (3.36). We later discuss the validity of these normalization constants (3.38), after introducing the 'unitarized model' in section 4 . The above choice is also regarded as specifying the weights of the summation over the winding sectors, which is equivalent to that over the interfaces along various cycles, as discussed shortly in the next subsection.

The total partition function is obtained by substituting these results (3.33), (3.34) and (3.38) into (3.8).

Let us finally comment on a useful rewriting of the relevant partition functions. If recalling the analysis for the simplest case $N=M=1$, the partition functions for the odd sectors are expected to be interpreted as the 'diagonal parts' of the even sectors. We here demonstrate this is indeed the case. Set

$$
Z_{\lambda}^{(0)}(\tau):=\sum_{\alpha \in S^{(N)}[\lambda]} \sum_{\tilde{\alpha} \in S^{(M)}[\lambda]} \tau_{\alpha}^{(N)} \widetilde{\tau}_{\tilde{\alpha}}^{(M)} \cdot Z_{R_{1}}(\tau)
$$

for ${ }^{\forall} \lambda \in \Lambda$. In terms of this,

$$
Z_{\lambda}^{\text {fiber }}(\tau)=Z_{\lambda}^{(0)}(\tau)^{2}
$$

for the even sector ${ }^{\forall} \lambda \in 2 \Lambda-\{0\}$. Moreover, the partition function (3.28) for the sector $\lambda=m \in 2 \mathbb{Z}+1$ with (3.38) is rewritten as

$$
Z_{m}^{\mathrm{fiber}}(\tau)=\mathbf{D}_{[m]} \cdot\left[Z_{2 m}^{(0)}(\tau)^{2}\right] \equiv Z_{2 m}^{(0)}(2 \tau)
$$

where $\mathbf{D}_{[\lambda]}$ denotes the operator extracting the diagonal part defined in (B.4) or (B.5) $([\lambda] \equiv \lambda \bmod 2 \Lambda)$. It is a straightforward task to define the 'diagonal part operator' $\mathbf{D}_{[\lambda]}$ $(\lambda \in \Lambda-2 \Lambda)$ to preserve the modular covariance, as is illustrated in appendix B. Thus, by the modular transformation, (3.41) extends to the case with general $\lambda \in \Lambda-2 \Lambda$ :

$$
Z_{\lambda}^{\text {fiber }}(\tau)=\mathbf{D}_{[\lambda]} \cdot\left[Z_{2 \lambda}^{(0)}(\tau)^{2}\right]
$$


In this way we can write down the total partition function in a compact form ${ }^{4}$

$$
\begin{aligned}
Z(\tau)= & (N M)^{2} Z_{0}^{\text {base }}(\tau) Z_{R_{1}}(\tau) Z_{R_{2}}(\tau)+\sum_{\lambda \in 2 \Lambda-\{0\}} Z_{\lambda}^{\text {base }}(\tau) Z_{\lambda}^{(0)}(\tau)^{2} \\
& +\sum_{\lambda \in \Lambda-2 \Lambda} Z_{\lambda}^{\text {base }}(\tau) \mathbf{D}_{[\lambda]} \cdot\left[Z_{2 \lambda}^{(0)}(\tau)^{2}\right]
\end{aligned}
$$

It is also easy to confirm that (3.43) actually reduces to the total partition function given in section 3.2 , when setting $N=M=1$.

\subsection{Interface operator $\mathcal{I}^{(-)}$along various cycles}

In our argument so far, $\lambda=w \tau+m \in \Lambda$ has represented the winding along the base circle. $^{5}$ From the point of view of the fiber sector, which is correlated to the base sector according to $\lambda$, one may also regard it as specifying the homology cycles of the world-sheet torus along which the interface lies. This would become evident, once we take the standard parameterization of the homology cycles so that they are labeled by $\gamma \in \Lambda(\equiv \mathbb{Z} \tau+\mathbb{Z})$ and the 'basic spatial (temporal) cycle' is associated with $\gamma=1(\gamma=\tau)$.

To be more specific, let us consider the interface along the cycle $\gamma \in \Lambda$ created by the operator $\mathcal{I}^{(-)}$in (3.5). We denote the corresponding fiber partition function with this interface inserted by ' $Z^{\text {fiber }}\left[\mathcal{I}_{\gamma}^{(-)}\right](\tau)$ '. It is quite natural to interpret $Z_{\lambda}^{\text {fiber }}(\tau)$ given in (3.33) and (3.34) to be this partition function for $\gamma=\lambda \in \Lambda$ up to the normalization factor $\mathcal{N}_{\lambda}$; namely,

$$
Z_{\lambda}^{\text {fiber }}(\tau) \equiv \mathcal{N}_{\lambda} Z^{\text {fiber }}\left[\mathcal{I}_{\lambda}^{(-)}\right](\tau)
$$

Indeed, for $\lambda=m \in \mathbb{Z}$, the left side reduces to (3.9), which represents the $m$-fold insertion of the interface operator along the basic spatial cycle. By the modular transformation, such a picture extends to the case of general $\lambda$. We take (3.44) to define the homology cycles for the interfaces in the following discussion.

With additional notation, the fiber partition function $Z^{\text {fiber }}\left[\mathcal{I}_{\lambda}^{(-)}\right](\tau)$ is written in a concise form. To this end, let us first introduce the subset $\Lambda_{0} \subset \Lambda$ defined by

$$
\Lambda_{0}:=\{1, \tau\} \cup\left\{n_{1} \tau+n_{2} ; n_{1} \in \mathbb{Z}_{>0}, n_{2} \in \mathbb{Z}-\{0\}, \operatorname{gcd}\left\{n_{1},\left|n_{2}\right|\right\}=1\right\} .
$$

Then, ${ }^{\forall} \gamma \in \Lambda$ can be uniquely expressible as $\gamma=n_{\gamma} \gamma_{0}, n_{\gamma} \in \mathbb{Z}, \gamma_{0} \in \Lambda_{0}$. We also introduce the 'polarized projection operators',

$$
\mathbf{P}_{\lambda}^{(N)}:=\frac{1}{N} \sum_{\alpha \in S^{(N)}[\lambda]} \tau_{\alpha}^{(N)}, \quad \widetilde{\mathbf{P}}_{\lambda}^{(M)}:=\frac{1}{M} \sum_{\tilde{\alpha} \in S^{(M)}[\lambda]} \widetilde{\tau}_{\tilde{\alpha}}^{(M)},
$$

\footnotetext{
${ }^{4}$ Since $S^{(K)}[\ell \lambda]=S^{(K)}[\lambda]\left({ }^{\forall} \ell \neq 0\right)$, one may replace $Z_{2 \lambda}^{(0)}(\tau)$ in (3.43) with $Z_{\lambda}^{(0)}(\tau)$. The above notation, however, makes it clear that the fiber partition functions for the odd sectors are regarded as the diagonal part of the even sectors. The 'unitarized model' discussed in section 4 is defined through $\widehat{S}^{(K)}[\lambda]$ in $(4.3)$, instead of $S^{(K)}[\lambda]$, and $\widehat{Z}_{2 \lambda}^{(0)}(\tau)$ is distinguished from $\widehat{Z}_{\lambda}^{(0)}(\tau)$ since $\widehat{S}^{(K)}[2 \lambda] \neq \widehat{S}^{(K)}[\lambda]$ generically.

${ }^{5}$ When $\lambda=w \tau+m \in \Lambda$ represents the winding of the base circle, $w$ and $m$ are the spatial and the temporal windings, respectively. They are related to the boundary condition of the base boson as $X(z+2 \pi i \gamma, \bar{z}-2 \pi i \bar{\gamma})=X(z, \bar{z})+2 \pi R_{\mathrm{b}}\langle\lambda, \gamma\rangle$ for any cycle $\gamma \in \Lambda$.
} 
where $S^{(K)}[\lambda]$ is defined in (3.32). Note that we can rewrite (3.46) as

$$
\mathbf{P}_{\lambda}^{(N)}=\frac{1}{N} \sum_{\alpha \in S^{(N)}[\lambda]} \tau_{M \alpha}^{(N)}, \quad \widetilde{\mathbf{P}}_{\lambda}^{(M)}=\frac{1}{M} \sum_{\tilde{\alpha} \in S^{(M)}[\lambda]} \widetilde{\tau}_{N \tilde{\alpha}}^{(M)},
$$

since $N$ and $M$ are coprime. This fact implies that the operators $\mathbf{P}_{\lambda}^{(N)}, \widetilde{\mathbf{P}}_{\lambda^{\prime}}^{(M)}$ commute with each other for ${ }^{\forall} \lambda, \lambda^{\prime} \in \Lambda$, which follows from the definitions of $\tau_{\gamma}^{\left({ }^{N}\right)}, \widetilde{\tau}_{\gamma}^{(N)}$ in (2.7), (2.10). The following identities are also obvious by definition,

$$
\mathbf{P}_{n \lambda}^{(N)}=\mathbf{P}_{\lambda}^{(N)}, \quad \widetilde{\mathbf{P}}_{n \lambda}^{(N)}=\widetilde{\mathbf{P}}_{\lambda}^{(N)},
$$

for ${ }^{\forall} n \in \mathbb{Z}$.

In terms of these polarized projection operators, (3.33), (3.34) and (3.42) are rephrased as follows:

even sectors: $\left(n_{\gamma} \in 2 \mathbb{Z}-\{0\}\right)$ :

$$
Z^{\text {fiber }}\left[\mathcal{I}_{\gamma}^{(-)}\right](\tau)=Z^{\text {fiber }}\left[\mathcal{I}_{2 \gamma_{0}}^{(-)}\right](\tau) \equiv\left[\mathbf{P}_{\gamma_{0}}^{(N)} \widetilde{\mathbf{P}}_{\gamma_{0}}^{(M)} \cdot Z_{R_{1}}(\tau)\right]^{2}
$$

odd sectors: $\left(n_{\gamma} \in 2 \mathbb{Z}+1\right)$ :

$$
Z^{\text {fiber }}\left[\mathcal{I}_{\gamma}^{(-)}\right](\tau)=Z^{\text {fiber }}\left[\mathcal{I}_{\gamma_{0}}^{(-)}\right](\tau) \equiv \mathbf{D}_{[\gamma]} \cdot Z^{\text {fiber }}\left[\mathcal{I}_{2 \gamma}^{(-)}\right](\tau)
$$

As in the case of $\lambda=m \in \mathbb{Z}$, the relevant cycles reduce down to $2 \gamma_{0}$ or $\gamma_{0}$, even when $\gamma$ is a multiple of them. By the definition (3.44), ' $\mathcal{I}_{2 \gamma_{0}}^{(-)}$', is also equivalent to the two-fold insertion of $\mathcal{I}_{\gamma_{0}}^{(-)}$along the 'short cycle' $\gamma_{0}$. These expressions are generalized later in section 4.3 .

\subsection{Relation to the ' $I^{(+)}$-twisted' models}

It would be a natural question what happens if we consider the models twisted by $I^{(+)}$in place of $I^{(-)}$. As mentioned in section $2.3, I^{(+)}$is obtained by taking the T-duality for the $\mathrm{CFT}_{1}$ as in (2.21). This means that

$$
\left[I^{(+)} \text {-model for the } S_{\tilde{R}_{1}}^{1} \times S_{R_{2}}^{1} \text {-fiber }\right] \stackrel{\text { T-duality }}{\cong}\left[I^{(-)} \text {-model for the } S_{R_{1}}^{1} \times S_{R_{2}}^{1} \text {-fiber }\right] \text {, }
$$

with $\tilde{R}_{1}=1 / R_{1}$ and $k_{1}=N, k_{2}=M$.

In the special case with $\tilde{R}_{1}=1 / R_{1}=R_{2}$ and $k_{1}=k_{2}=1$, the interface operator $\mathcal{I}^{(+)}$ composed of $I^{(+)}$becomes the permutation operator $\mathcal{P}$. Therefore, the $I^{(-)}$-model for the $S_{1 / R_{2}}^{1} \times S_{R_{2}}^{1}$-fiber can be interpreted as the T-dual of the 'permutation twisted model', that is, the orbifold of $S_{R_{\mathrm{b}}}^{1} \times\left[S_{R_{2}}^{1} \times S_{R_{2}}^{1}\right]$ twisted by

$$
\mathcal{T}_{2 \pi R_{\mathrm{b}}} \otimes \mathcal{P}
$$

Along the fiber direction, this indeed acts as the permutation $\left(X_{1}, X_{2}\right) \mapsto\left(X_{2}, X_{1}\right)$. 
Let us evaluate the partition function of this permutation orbifold. It is convenient to introduce the new coordinates,

$$
X_{ \pm}:=\frac{1}{\sqrt{2}}\left(X_{1} \pm X_{2}\right) .
$$

Then, the permutation $\mathcal{P}$ acts as

$$
\mathcal{P}: X_{ \pm} \longmapsto \pm X_{ \pm}
$$

Namely, $\mathcal{P}$ acts as the ordinary reflection orbifold with respect to the $X_{-}$-direction. The insertion of $\mathcal{P}$ into the trace only leaves the Fock vacua with

$$
p_{-, L} \equiv \frac{1}{\sqrt{2}}\left(p_{1, L}-p_{2, L}\right)=0, \quad p_{-, R} \equiv \frac{1}{\sqrt{2}}\left(p_{1, R}-p_{2, R}\right)=0 .
$$

On the other hand, the $X_{+}$-direction is compactified onto the circle with the radius $\sqrt{2} R_{2}$, but the zero-mode spectrum is constrained as

$$
\text { KK momentum } \in 2 \mathbb{Z}, \quad \text { winding } \in \mathbb{Z},
$$

due to the condition (3.54). Thus, we eventually obtain the fiber partition function for the sector $\lambda \equiv m \in 2 \mathbb{Z}+1$,

$$
\begin{aligned}
Z_{\lambda}^{\text {fiber }}(\tau) & =\left|\frac{2 \eta(\tau)}{\theta_{2}(\tau)}\right| \frac{1}{|\eta(\tau)|^{2}} \sum_{r, s \in \mathbb{Z}} q^{\frac{1}{4}\left(\frac{2 r}{\sqrt{2} R_{2}}+\sqrt{2} R_{2} s\right)^{2}} q^{\frac{1}{4}\left(\frac{2 r}{\sqrt{2} R_{2}}-\sqrt{2} R_{2} s\right)^{2}} \\
& \equiv\left|\frac{2 \eta(\tau)}{\theta_{2}(\tau)}\right| \sum_{\nu \in \mathbb{Z} \tau+\frac{1}{2} \mathbb{Z}} Z_{\sqrt{2} R_{2}}(\tau \mid \nu) .
\end{aligned}
$$

We also obtain

$$
Z_{\lambda}^{\text {fiber }}(\tau)=Z_{R_{2}}(\tau)^{2}
$$

for $\lambda=m \in 2 \mathbb{Z}$, since $\mathcal{P}^{2}=\mathbf{1}$.

Based on these results and the modular invariance, we can uniquely determine the fiber partition functions $Z_{\lambda}^{\text {fiber }}(\tau)$ with ${ }^{\forall} \lambda \in \Lambda$, which are equal to those of the $I^{(-)}$-model with $R_{1}=1 / R_{2}$ and $N=M=1$, as is anticipated. In this way, we have seen that the simplest case $N=M=1$ reduces to a geometrically realized model, that is, the orbifold by (3.51). A similar construction of the partition function involving the permutation is given in [9]. However, in generic cases $N M>1$, our models would be still non-geometric even in the $I^{(+)}$-picture, because the gluing condition (2.25) non-trivially restricts both of the KK and the winding spectra.

\section{Unitarized models}

Let us discuss the unitarity of the modular invariant models we constructed in the previous section. The $N=M=1$ model obviously leads to a unitary spectrum as in ordinary $\mathbb{Z}_{2^{-}}$ orbifolds. However, in the cases of $N M>1$, the spectrum gets non-unitary. For example, 
one can observe a non-unitary $q$-expansion in the sector with base winding $w=N,{ }^{6}$ that is,

$$
\lambda=N \tau+m, \quad(m \in \mathbb{Z}),
$$

except for the $N=1$ case. In fact, we find $S^{(N)}[N \tau]=\mathbb{Z}_{N} \tau$, while $S^{(N)}[N \tau+m]=\mathbb{Z}_{N}$ holds if $m \neq 0$ and $\operatorname{gcd}\{|m|, N\}=1$. Thus, it is not difficult to see that, in this situation, we never achieve the $q$-expansion with positive coefficients for the $w=N$ sector, after making the Poisson resummation with respect to $m \in \mathbb{Z}$.

\subsection{Definition of the unitarized model}

Therefore, we shall propose the 'unitarized' model for the $N M>1$ cases. Though the complete solution to the unitarization is an important future problem, one can find a simple and interesting solution which is interpreted in terms of the discrete torsion $[30,31]$ and multiple insertions of the interface operators. Indeed, it turns out that one can construct a simple unitary extension of the present model at least in the cases,

$$
R_{1}=R_{2}=\sqrt{\frac{N}{M}}=: R_{\mathrm{f}}, \quad \operatorname{gcd}\{N, M\}=1,
$$

which satisfy the condition (2.15). We would like to discuss more general cases with generic $R_{1}$ and $R_{2}$ satisfying (2.15) in the future work.

Let us proceed with the assumption (4.2). Our construction of the unitarized model is simple. All we have to do is to replace the set $S^{(K)}[\lambda]$ defined in (3.32) with

$$
\widehat{S}^{(K)}[\lambda]:=\{\nu \in \Lambda ;\langle\lambda, \nu\rangle \in K \mathbb{Z}\} / K \Lambda,
$$

in (3.39), (3.40) and (3.42). Namely, we replace (3.39) with

$$
\widehat{Z}_{\lambda}^{(0)}(\tau):=\sum_{\alpha \in \widehat{S}^{(N)}[\lambda]} \sum_{\tilde{\alpha} \in \widehat{S}^{(M)}[\lambda]} \tau_{\alpha}^{(N)} \widetilde{\tau}_{\tilde{\alpha}}^{(M)} \cdot Z_{R_{\mathrm{f}}}(\tau),
$$

and the fiber partition functions $Z_{\lambda}^{\text {fiber }}(\tau)$ with the following: ${ }^{7}$

even sectors: $\lambda \in 2 \Lambda$ :

$$
\widehat{Z}_{\lambda}^{\text {fiber }}(\tau)=\widehat{Z}_{\lambda}^{(0)}(\tau)^{2}
$$

\footnotetext{
${ }^{6}$ Since we are considering the twisted compactification due to (3.4), which includes the shift operator $\mathcal{T}_{2 \pi R_{\mathrm{b}}}$, we should regard the sectors with $w \neq 0$ as the 'twisted sectors' in the context of orbifold theory, whereas the temporal winding $m$ has to be dualized into the KK momentum in order to read off the spectrum.

${ }^{7}$ If we make the replacement (4.3) in (3.34), instead of (3.42), it is subtle if the resultant expression in general becomes equal to $(4.6)$ : when $\operatorname{gcd}\{2 N, M\}=2, \tau_{\alpha}^{(2 N)} \widetilde{\tau}_{\tilde{\alpha}}^{(M)}$ may not necessarily be replaced with $\tau_{M \alpha}^{(2 N)} \widetilde{\tau}_{2 N \tilde{\alpha}}^{(M)}$ for $\alpha \in \widehat{S}^{(2 N)}[\lambda], \tilde{\alpha} \in \widehat{S}^{(M)}[\lambda]$, and hence not commute. Even in that case, the expression (4.6) is well-defined.
} 
odd sectors: $\lambda \in \Lambda-2 \Lambda$ :

$$
\widehat{Z}_{\lambda}^{\text {fiber }}(\tau)=\mathbf{D}_{[\lambda]} \cdot\left[\widehat{Z}_{2 \lambda}^{(0)}(\tau)^{2}\right]
$$

The $\lambda=0$ case has been combined into (4.5), since $\widehat{S}^{(N)}[0]=\Lambda / N \Lambda, \widehat{S}^{(M)}[0]=\Lambda / M \Lambda$ and thus

$$
\widehat{Z}_{Z^{\text {fiber }}} 0(\tau)=(N M)^{2} Z_{\frac{M}{N} R_{\mathrm{f}}}(\tau)^{2} \equiv(N M)^{2} Z_{1 / R_{\mathrm{f}}}(\tau)^{2} \equiv(N M)^{2} Z_{R_{\mathrm{f}}}(\tau)^{2} .
$$

Consequently, the total partition function of the unitarized model is defined by

$$
\widehat{Z}(\tau):=\sum_{\lambda \in 2 \Lambda} Z_{\lambda}^{\mathrm{base}}(\tau) \widehat{Z}_{\lambda}^{(0)}(\tau)^{2}+\sum_{\lambda \in \Lambda-2 \Lambda} Z_{\lambda}^{\mathrm{base}}(\tau) \mathbf{D}_{[\lambda]} \cdot\left[\widehat{Z}_{2 \lambda}^{(0)}(\tau)\right]^{2} .
$$

Again one can easily check that this function reduces to the total partition function given in section 3.2, when setting $N=M=1$.

From the definition, it follows that $S^{(K)}[\lambda] \subset \widehat{S}^{(K)}[\lambda]$ in general, and that

$$
S^{(K)}[\lambda]=\widehat{S}^{(K)}[\lambda], \quad \text { iff } \operatorname{gcd}\{|w|,|m|, K\}=1, \quad(\lambda \equiv w \tau+m \in \Lambda), \quad \text { or } \lambda=0 .
$$

In other words, new twisted sectors are created when $\operatorname{gcd}\{|w|,|m|, K\}>1$. We note that $\widehat{S}^{(K)}[\lambda]$ has a periodicity,

$$
\widehat{S}^{(K)}\left[\lambda+K \lambda^{\prime}\right]=\widehat{S}^{(K)}[\lambda], \quad\left({ }^{\forall} \lambda^{\prime} \in \Lambda\right) .
$$

As mentioned above and discussed below, the replacement by $\widehat{S}^{(K)}[\lambda]$ is interpretable in terms of the discrete torsion in the theory of orbifolds, which is expected to preserve the unitarity. This fact also motivated us to define the unitarized model in such a way. Furthermore, we will discuss later how the new partition function $\widehat{Z^{\mathrm{fiber}}}{ }_{\lambda}(\tau)$ is interpreted to be generated by multiple insertions of $\mathcal{I}^{(-)}$along various cycles of the world-sheet torus.

\subsection{Proof of unitarity}

We here demonstrate that the proposed model given by (4.8) is unitary. Namely, we show that the total partition function (4.8) is indeed $q$-expanded only with positive integral coefficients, after making the Poisson resummation with respect to $m \in \mathbb{Z}$ in $\lambda \equiv w \tau+m$.

We proceed by three steps: first, since the total partition function looks quite intricate, we analyze a simpler part of the partition function in (4.4). Second, using the result in the first step, we show that the sectors with even $w$ in $\lambda=w \tau+m$ have a $q$-expansion with positive integral coefficients. Finally, we show a similar statement for the sectors with odd $w$.

(i) evaluation of $\widehat{Z}_{\lambda}^{(0)}(\tau)$ : we first examine the simpler partition function $\widehat{Z}_{\lambda}^{(0)}(\tau)$ defined in (4.4). With this aim it is convenient to introduce the phase factor defined by

$$
\begin{aligned}
\epsilon_{j}^{(K)}(\lambda, \gamma ; \tau):= & e^{-2 \pi i \frac{j}{K}\langle\lambda, \gamma\rangle_{\tau}} \equiv e^{-2 \pi i \frac{j}{K}(w b-m a)}, \\
& \left(j \in \mathbb{Z}_{K}, \lambda \equiv w \tau+m \in \Lambda, \gamma \equiv a \tau+b \in \Lambda\right),
\end{aligned}
$$


where $\langle,\rangle_{\tau}$ is defined in (2.11) (see also appendix A). By the subscript, we have made the $\tau$-dependence explicit to avoid confusion. Since

$$
\frac{1}{K} \sum_{j \in \mathbb{Z}_{K}} \epsilon_{j}^{(K)}(\lambda, \gamma ; \tau)= \begin{cases}1 & \langle\lambda, \gamma\rangle_{\tau} \in K \mathbb{Z} \\ 0 & \text { otherwise }\end{cases}
$$

we can rewrite $\widehat{Z}_{\lambda}^{(0)}(\tau)$ as

$$
\widehat{Z}_{\lambda}^{(0)}(\tau)=\sum_{j \in \mathbb{Z}_{N}} \sum_{\tilde{j} \in \mathbb{Z}_{M}} \widehat{Z}_{\lambda,[j, \tilde{j}]}^{(0)}(\tau)
$$

with

$$
\begin{aligned}
\widehat{Z}_{\lambda,[j, \tilde{j}]}^{(0)}(\tau) & :=\frac{1}{N M} \sum_{\alpha \in \Lambda / N \Lambda} \sum_{\tilde{\alpha} \in \Lambda / M \Lambda} \epsilon_{j}^{(N)}(\lambda, \alpha ; \tau) \epsilon_{\tilde{j}}^{(M)}(\lambda, \tilde{\alpha} ; \tau) \tau_{\alpha}^{(N)} \widetilde{\tau}_{\tilde{\alpha}}^{(M)} \cdot Z_{R_{\mathrm{f}}}(\tau) \\
& \equiv \frac{1}{N M} \sum_{\alpha \in \Lambda / N \Lambda} \sum_{\tilde{\alpha} \in \Lambda / M \Lambda} \epsilon_{j}^{(N)}(\lambda, M \alpha ; \tau) \epsilon_{\tilde{j}}^{(M)}(\lambda, N \tilde{\alpha} ; \tau) \tau_{M \alpha}^{(N)} \widetilde{\tau}_{N \tilde{\alpha}}^{(M)} \cdot Z_{R_{\mathrm{f}}}(\tau)
\end{aligned}
$$

In the second line, we made use of the assumption $\operatorname{gcd}\{N, M\}=1$. Note here that $\tau_{M \alpha}^{(N)}$ and $\widetilde{\tau}_{N \tilde{\alpha}}^{(M)}$ always commute with each other, while $\tau_{\alpha}^{(N)}$ and $\widetilde{\tau}_{\tilde{\alpha}}^{(M)}$ do not.

The phase factor $\epsilon_{j}^{(N)}(\lambda, \gamma ; \tau) \epsilon_{\tilde{j}}^{(M)}(\lambda, \gamma ; \tau)$ is interpreted as the discrete torsion [30, 31], as we addressed before. Actually, it turns out that the sector of the partition function (4.8) with $w$ even or odd separately leads to a unitary $q$-expansion. To see this below, it is important to observe that (4.14) is explicitly evaluated as

$$
\begin{aligned}
\widehat{Z}_{\lambda=w \tau+m,[j, \tilde{j}]}^{(0)}(\tau)=\sum_{a \in \mathbb{Z}_{N}} \sum_{\tilde{a} \in \mathbb{Z}_{M}} \sum_{r \in \mathbb{Z}_{2}} e^{2 \pi i m\left(\frac{j M a}{N}+\frac{\tilde{j} N \tilde{a}}{M}\right)} & \times Z^{(N M)}[M j w+N \tilde{a}+N M r, N \tilde{j} w+M a](\tau) .
\end{aligned}
$$

Here, we set $\alpha=a \tau+b, \tilde{\alpha}=\tilde{a} \tau+\tilde{b}$, summed over $b \in \mathbb{Z}_{N}, \tilde{b} \in \mathbb{Z}_{M}$ and used the notation (2.6). This expression is suited for making the Poisson resummation, since the temporal winding $m$ appears only in the phase factor $e^{2 \pi i m\left(\frac{j M a}{N}+\frac{\tilde{j} N \tilde{a}}{M}\right)}$.

(ii) unitarity of $w \in 2 \mathbb{Z}$ sectors: now, we are ready to show the unitarity of the model or the partition function in question, that is, $\widehat{Z}(\tau)$ in (4.8). We first focus on the case of $\lambda=w \tau+m$ with a fixed value $w \in 2 \mathbb{Z}$, and consider the Poisson resummation with respect to $m$.

For the cases of $m \in 2 \mathbb{Z}$, the fiber partition function is equal to the square of (4.4), that is,

$$
\widehat{Z_{\lambda}^{\text {fiber }}}{ }_{\lambda}(\tau)=\prod_{i=1,2} \sum_{j_{i} \in \mathbb{Z}_{N}} \sum_{\tilde{j}_{i} \in \mathbb{Z}_{M}} \widehat{Z}_{\lambda,\left[j_{i}, \tilde{j}_{i}\right]}(\tau) .
$$

Then, it is straightforward to make the Poisson resummation over $m \in 2 \mathbb{Z}$ with the help of (4.15). Note that the phase factor appearing in (4.15) just shifts the KK momentum along the base circle as

$$
\frac{n}{2 R_{\mathrm{b}}} \longrightarrow \frac{1}{2 R_{\mathrm{b}}}\left[n+2 \sum_{i=1,2}\left(\frac{j_{i} M a_{i}}{N}+\frac{\tilde{j}_{i} N \tilde{a}_{i}}{M}\right)\right],
$$


where $i=1,2$ labels the contributions from the two factors of $\widehat{Z}_{\lambda}^{(0)}(\tau)$. We thus obtain

$$
\begin{aligned}
\widehat{Z}_{\text {even }, w}(\tau) & \equiv \sum_{m \in 2 \mathbb{Z}} Z_{w \tau+m}^{\text {base }}(\tau) \widehat{Z^{\text {fiber }}} w \tau+m(\tau) \\
= & \sum_{j_{i}, a_{i} \in \mathbb{Z}_{N}} \sum_{\tilde{j}_{i}, \tilde{a}_{i} \in \mathbb{Z}_{M}} \sum_{r_{i} \in \mathbb{Z}_{2}} \sum_{n \in \mathbb{Z}}\left[\prod_{i=1,2} Z^{(N M)}\left[M j_{i} w+N \tilde{a}_{i}+N M r_{i}, N \tilde{j}_{i} w+M a_{i}\right](\tau)\right] \\
& \times \frac{1}{2} \frac{1}{|\eta(\tau)|^{2}} q^{\frac{1}{4}\left[\frac{1}{2 R_{\mathrm{b}}}\left\{n+2 \sum_{i=1,2}\left(\frac{j_{i} M a_{i}}{N}+\frac{\tilde{j}_{i} N \tilde{a}_{i}}{M}\right)\right\}+R_{\mathrm{b}} w\right]^{2}} \\
& \times q^{\frac{1}{4}\left[\frac{1}{2 R_{\mathrm{b}}}\left\{n+2 \sum_{i=1,2}\left(\frac{j_{i} M a_{i}}{N}+\frac{\tilde{j}_{i} N \tilde{a}_{i}}{M}\right)\right\}-R_{\mathrm{b}} w\right]^{2}} .
\end{aligned}
$$

It is not difficult to see that the sectors with 'non-diagonal' quantum numbers $\left(j_{1}, a_{1}, \ldots\right) \neq\left(j_{2}, a_{2}, \ldots\right)$ are expanded in a $q$-series with positive integral coefficients. Note that the factor $\frac{1}{2}$ appearing in (4.18) indeed cancels out due to the degeneracy of relevant contributions. Furthermore, the partition functions for the 'diagonal' sectors with $j_{1}=j_{2} \equiv j, a_{1}=a_{2} \equiv a$, and so on, are rewritten by using the product formula of theta function (A.8) as

$$
\begin{aligned}
\widehat{Z}_{\text {even }, w}[j, \tilde{j}, a, \tilde{a}, r](\tau)= & \frac{1}{|\eta(\tau)|^{2}} Z^{(2 N M)}[2 M j w+2 N \tilde{a}+2 N M r, 2 N \tilde{j} w+2 M a](\tau) \\
& \times \frac{1}{2} \sum_{n \in \mathbb{Z}} \frac{1}{|\eta(\tau)|^{2}} q^{\frac{1}{4}\left[\frac{1}{2 R_{\mathrm{b}}}\left\{n+4\left(\frac{j M a}{N}+\frac{\tilde{j} N \tilde{a}}{M}\right)\right\}+R_{\mathrm{b}} w\right]^{2}} \\
& \times q^{\frac{1}{4}\left[\frac{1}{2 R_{\mathrm{b}}}\left\{n+4\left(\frac{j M a}{N}+\frac{\tilde{j} N \tilde{a}}{M}\right)\right\}-R_{\mathrm{b}} w\right]^{2}} \\
& +[\text { residual terms], }
\end{aligned}
$$

The 'residual terms' in the above denote some $q$-series with positive integral coefficients whose explicit form is not important here.

On the other hand, by using the definition of $\mathbf{D}_{[\lambda]}$ given in (B.5), we obtain for $m \in 2 \mathbb{Z}+1$,

$$
\begin{aligned}
\widehat{Z^{\text {fiber }}}{ }_{\lambda}(\tau)= & \mathbf{D}_{[\lambda]} \cdot\left[\prod_{i=1,2} \sum_{j_{i} \in \mathbb{Z}_{N}} \sum_{\tilde{j}_{i} \in \mathbb{Z}_{M}} \widehat{Z}_{2 \lambda,\left[j_{i}, \tilde{j}_{i}\right]}(\tau)\right] \\
= & \sum_{j \in \mathbb{Z}_{N}} \sum_{\tilde{j} \in \mathbb{Z}_{M}} \widehat{Z}_{2 \lambda,[j, \tilde{j}]}^{(0)}(2 \tau) \\
= & \sum_{j \in \mathbb{Z}_{N}} \sum_{\tilde{j} \in \mathbb{Z}_{M}} \sum_{a \in \mathbb{Z}_{N}} \sum_{\tilde{a} \in \mathbb{Z}_{M}} \sum_{r \in \mathbb{Z}_{2}} e^{2 \pi i m \cdot 2\left(\frac{j M a}{N}+\frac{\tilde{j} N \tilde{a}}{M}\right)} \\
& \times Z^{(N M)}[M j w+N \tilde{a}+N M r, N \tilde{j} w+M a](2 \tau) \\
= & \sum_{j \in \mathbb{Z}_{N}} \sum_{\tilde{j} \in \mathbb{Z}_{M}} \sum_{a \in \mathbb{Z}_{N}} \sum_{\tilde{a} \in \mathbb{Z}_{M}} \sum_{r \in \mathbb{Z}_{2}} e^{2 \pi i m \cdot 2\left(\frac{j M a}{N}+\frac{\tilde{j} N \tilde{a}}{M}\right)} \\
& \quad \times\left|\frac{2 \eta(\tau)}{\theta_{2}(\tau)}\right| Z^{(2 N M)}[2 M j w+2 N \tilde{a}+2 N M r, 2 N \tilde{j} w+2 M a](\tau) .
\end{aligned}
$$


Note here that the operator $\mathbf{D}_{[\lambda]}$ for this sector acts as $\tau \rightarrow 2 \tau$, while keeping $\lambda \equiv w \tau+m$ intact. This implies that

$$
\begin{aligned}
\epsilon_{j}^{(N)}(2 \lambda, M\{a(2 \tau) & +b\} ; 2 \tau) \epsilon_{\tilde{j}}^{(M)}(2 \lambda, N\{\tilde{a}(2 \tau)+\tilde{b}\} ; 2 \tau) \\
& =e^{-2 \pi i \frac{j M}{N}\langle w(2 \tau)+2 m, a(2 \tau)+b\rangle_{2 \tau}} e^{-2 \pi i \frac{\tilde{j} N}{M}\langle w(2 \tau)+2 m, \tilde{a}(2 \tau)+\tilde{b}\rangle_{2 \tau}} \\
& =e^{-2 \pi i \frac{j M}{N}(w b-2 m a)} e^{-2 \pi i \frac{\tilde{j} N}{M}(w \tilde{b}-2 m \tilde{a})}
\end{aligned}
$$

which explains the phase factor appearing in (4.20). As a consistency check, we can confirm the modular T-invariance of (4.20),

$$
\widehat{Z}_{\lambda}^{\text {fiber }}{ }_{\lambda}(\tau+1)={\widehat{Z^{\text {fiber }}}}_{\lambda}(\tau), \quad\left({ }^{\forall} \lambda \equiv w \tau+m, w \in 2 \mathbb{Z}, m \in 2 \mathbb{Z}+1\right) .
$$

The expressions (4.20) is again suitable for the Poisson resummation. Namely, the summation over $m \in 2 \mathbb{Z}+1$ is evaluated as

$$
\begin{aligned}
\widehat{Z}_{\text {odd }, w}(\tau) & \equiv \sum_{m \in 2 \mathbb{Z}+1}{\widehat{Z^{\text {fiber }}}}_{w \tau+m}(\tau) Z_{w \tau+m}^{\text {base }}(\tau) \\
& \equiv \sum_{j, a \in \mathbb{Z}_{N}} \sum_{\tilde{j}, \tilde{a} \in \mathbb{Z}_{M}} \sum_{r \in \mathbb{Z}_{2}} \widehat{Z}_{\text {odd }, w}[j, \tilde{j}, a, \tilde{a}, r](\tau),
\end{aligned}
$$

with

$$
\begin{aligned}
& \widehat{Z}_{\text {odd }, w} {[j, \tilde{j}, a, \tilde{a}, r](\tau)=\left|\frac{2 \eta(\tau)}{\theta_{2}(\tau)}\right| Z^{(2 N M)}[2 M j w+2 N \tilde{a}+2 N M r, 2 N \tilde{j} w+2 M a](\tau) } \\
& \times \sum_{n \in \mathbb{Z}} \frac{1}{2} \frac{(-1)^{n}}{|\eta(\tau)|^{2}} q^{\frac{1}{4}\left[\frac{1}{2 R_{\mathrm{b}}}\left\{n+4\left(\frac{j M a}{N}+\frac{\tilde{j} N \tilde{a}}{M}\right)\right\}+R_{\mathrm{b}} w\right]^{2}} \overline{q^{\frac{1}{4}\left[\frac{1}{2 R_{\mathrm{b}}}\left\{n+4\left(\frac{j M a}{N}+\frac{\tilde{j} N \tilde{a}}{M}\right)\right\}-R_{\mathrm{b}} w\right]^{2}}} .
\end{aligned}
$$

Comparing (4.19) and (4.24), it is now obvious that the summation

$$
\widehat{Z}_{\text {even }, w}[j, \tilde{j}, a, \tilde{a}, r](\tau)+\widehat{Z}_{\mathrm{odd}, w}[j, \tilde{j}, a, \tilde{a}, r](\tau),
$$

is written in a unitary $q$-series for each $j, \tilde{j}, a, \tilde{a}, r$.

(iii) unitarity of $w \in 2 \mathbb{Z}+1$ sectors: let us examine the remaining cases with $w \in 2 \mathbb{Z}+$ 1. By means of (B.5), we obtain the following:

- For $m \in 2 \mathbb{Z}$,

$$
\begin{aligned}
\widehat{Z_{\lambda}^{\mathrm{fiber}}}{ }_{\lambda}(\tau)= & \mathbf{D}_{[\lambda]} \cdot\left[\prod_{i=1,2} \sum_{j_{i} \in \mathbb{Z}_{N}} \sum_{\tilde{j}_{i} \in \mathbb{Z}_{M}} \widehat{Z}_{2 \lambda,\left[j_{i}, \tilde{j}_{i}\right]}(\tau)\right] \\
= & \sum_{j \in \mathbb{Z}_{N}} \sum_{\tilde{j} \in \mathbb{Z}_{M}} \widehat{Z}_{\frac{1}{2}(2 \lambda),[j, \tilde{j}]}^{(0)}\left(\frac{\tau}{2}\right) \\
= & \sum_{j \in \mathbb{Z}_{N}} \sum_{\tilde{j} \in \mathbb{Z}_{M}} \sum_{a \in \mathbb{Z}_{N}} \sum_{\tilde{a} \in \mathbb{Z}_{M}} \sum_{r \in \mathbb{Z}_{2}} e^{2 \pi i m\left(\frac{j M a}{N}+\frac{\tilde{j} N \tilde{a}}{M}\right)} \\
& \times Z^{(N M)}[2 M j w+N \tilde{a}+N M r, 2 N \tilde{j} w+M a]\left(\frac{\tau}{2}\right) .
\end{aligned}
$$


- For $m \in 2 \mathbb{Z}+1$,

$$
\begin{aligned}
\widehat{Z_{\lambda}^{\text {fiber }}}{ }_{\lambda}(\tau)= & \mathbf{D}_{[\lambda]} \cdot\left[\prod_{i=1,2} \sum_{j_{i} \in \mathbb{Z}_{N}} \sum_{\tilde{j}_{i} \in \mathbb{Z}_{M}} \widehat{Z}_{2 \lambda,\left[j_{i}, \tilde{j}_{i}\right]}(\tau)\right] \\
= & \sum_{j \in \mathbb{Z}_{N}} \sum_{\tilde{j} \in \mathbb{Z}_{M}} \widehat{Z}_{\frac{1}{2}(2 \lambda),[j, \tilde{j}]}^{(0)}\left(\frac{\tau+1}{2}\right) \\
= & \sum_{j \in \mathbb{Z}_{N}} \sum_{\tilde{j} \in \mathbb{Z}_{M}} \sum_{a \in \mathbb{Z}_{N}} \sum_{\tilde{a} \in \mathbb{Z}_{M}} \sum_{r \in \mathbb{Z}_{2}} e^{2 \pi i(m-w)\left(\frac{j M a}{N}+\frac{\tilde{j} N \tilde{a}}{M}\right)} \\
& \times Z^{(N M)}[2 M j w+N \tilde{a}+N M r, 2 N \tilde{j} w+M a]\left(\frac{\tau+1}{2}\right) .
\end{aligned}
$$

In these evaluations, the phase factors were slightly non-trivial again. In (4.26), for example, we made the following evaluation,

$$
\begin{aligned}
\epsilon_{j}^{(N)}\left(\lambda, M\left\{a \frac{\tau}{2}+b\right\} ; \frac{\tau}{2}\right) & \epsilon_{\tilde{j}}^{(M)}\left(\lambda, N\left\{\tilde{a} \frac{\tau}{2}+\tilde{b}\right\} ; \frac{\tau}{2}\right) \\
= & e^{-2 \pi i \frac{j M}{N}\left\langle 2 w \frac{\tau}{2}+m, a \frac{\tau}{2}+b\right\rangle_{\frac{\tau}{2}}} e^{-2 \pi i \frac{\tilde{j} N}{M}\left\langle 2 w \frac{\tau}{2}+m, \tilde{a} \frac{\tau}{2}+\tilde{b}\right\rangle_{\frac{\tau}{2}}} \\
= & e^{-2 \pi i \frac{j M}{N}(2 w b-m a)} e^{-2 \pi i \frac{j N}{M}(2 w \tilde{b}-m \tilde{a})}
\end{aligned}
$$

If only picking (4.26) up, we are clearly led to a unitary $q$-series by Poisson resumming over $m \in 2 \mathbb{Z}$, as in the case of $\widehat{Z}_{\text {even, } w}(\tau)$ in (4.18). Moreover, as expected, (4.26) and (4.27) are combined into a T-invariant form, since

$$
{\widehat{Z^{\text {fiber }}}}_{\lambda}(\tau+1)={\widehat{Z^{\text {fiber }}}}_{\lambda}(\tau), \quad\left(\lambda \equiv w \tau+m,{ }^{\forall} w \in 2 \mathbb{Z}+1,{ }^{\forall} m \in \mathbb{Z}\right) .
$$

This means that the summation over $m \in 2 \mathbb{Z}+1$ is written in the same form of $q$-series as that for $m \in 2 \mathbb{Z}$, but with an extra sign \pm 1 in each term. Therefore, the partition function

$$
\begin{aligned}
& \sum_{m \in \mathbb{Z}} \widehat{Z}^{\text {fiber }}{ }_{w \tau+m}(\tau) Z_{w \tau+m}^{\text {base }}(\tau)
\end{aligned}
$$

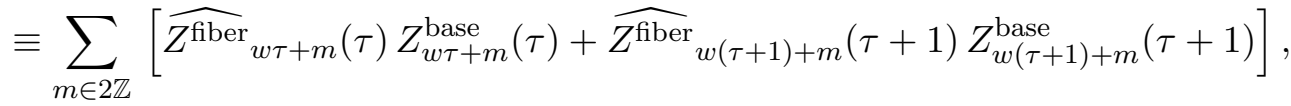

again produces a unitary $q$-series. Similarly to the twisted sectors of the familiar $\mathbb{Z}_{2^{-}}$ orbifolds, the negative terms due to the oscillator part cancel with the corresponding positive terms.

In this way, we have succeeded in showing the unitarity of the proposed model (4.8). We add a few comments:

- It is worthwhile to point out that there exists a unique vacuum with minimal conformal weights $h=\tilde{h}=0$ (the 'identity state') in the spectrum read off from the partition function (4.25) for the sector $w=j=\tilde{j}=a=\tilde{a}=r=0$. This fact would suggest that our choice of the normalization constants (3.38) is reasonable. 
- As clarified in the above discussion, the even sectors with $\lambda \in 2 \Lambda$ are reinterpreted as the orbifold with the discrete torsion. Nevertheless, the total partition function (4.8) is not likely to be described by any model of orbifold in the precise sense, except for the simplest case $N=M=1$. This is because the operator $\mathbf{D}_{[\lambda]}$ is hard to be explicitly realized in terms of an automorphism acting on the Hilbert space of CFT.

\subsection{Interpretation of the unitarized model: multiple insertions of the interface operators}

At the last of this section we would like to discuss a possible physical interpretation of the unitarized model described by (4.8), or the fiber partition functions $\widehat{Z^{\text {fiber }}}{ }_{\lambda}(\tau)$ given in (4.5) and (4.6). Let us start by extending the formulas (3.49), (3.50) to the cases of multiple insertions of the interface operator $\mathcal{I}^{(-)}$along different cycles,

$$
\begin{gathered}
Z^{\text {fiber }}\left[\mathcal{I}_{\gamma_{1}}^{(-)}, \ldots, \mathcal{I}_{\gamma_{\ell}}^{(-)}\right](\tau):=\left[\left(\prod_{j=1}^{\ell} \mathbf{P}_{\gamma_{j}}^{(N)} \widetilde{\mathbf{P}}_{\gamma_{j}}^{(M)}\right) \cdot Z_{\sqrt{\frac{N}{M}}}(\tau)\right]^{2}, \quad\left(\gamma_{1}, \ldots, \gamma_{\ell} \in 2 \Lambda-\{0\}\right), \\
Z^{\text {fiber }}\left[\mathcal{I}_{\gamma}^{(-)}, \mathcal{I}_{\gamma_{1}}^{(-)}, \ldots, \mathcal{I}_{\gamma_{\ell}}^{(-)}\right](\tau):=\mathbf{D}_{[\gamma]} \cdot\left[\begin{array}{c}
\left.\mathbf{P}_{2 \gamma}^{(N)} \widetilde{\mathbf{P}}_{2 \gamma}^{(M)}\left(\prod_{j=1}^{\ell} \mathbf{P}_{\gamma_{j}}^{(N)} \widetilde{\mathbf{P}}_{\gamma_{j}}^{(M)}\right) \cdot Z_{\sqrt{\frac{N}{M}}}(\tau)\right]^{2}, \\
\left(\gamma \in \Lambda-2 \Lambda, \gamma_{1}, \ldots, \gamma_{\ell} \in 2 \Lambda-\{0\}\right) .
\end{array}\right.
\end{gathered}
$$

These expressions are well-defined because the operators $\mathbf{P}_{*}^{(N)}, \widetilde{\mathbf{P}}_{*}^{(M)}$ commute with one another, as we already mentioned. One should keep it in mind that all the operators $\tau_{*}^{(N)}, \widetilde{\tau}_{*}^{(M)}$ implicitly appearing in $(4.31),(4.32)$ have to be interpreted as $\tau_{*}^{(N), R_{\mathrm{f}}}, \widetilde{\tau}_{*}^{(M), R_{\mathrm{f}}}$ respectively. At present, we do not have a proper extension for any two insertions along different odd cycles; $\gamma_{i}, \gamma_{j} \in \Lambda-2 \Lambda, \gamma_{i} \neq \gamma_{j}$.

Now, let us focus on the partition functions $\widehat{Z^{\text {fiber }}}{ }_{\lambda}(\tau)$ given in (4.5), (4.6). We attempt to rewrite them in the forms of (4.31) and (4.32). Recall that the partition function in the even sector (4.5) is obtained by replacing the sets $S^{(*)}[\lambda]$ appearing in (3.33) with their 'hatted' counterparts (4.3), and in the odd sector by acting with the diagonal operator $\mathbf{D}_{[\lambda]}$.

We again begin our analysis with the decomposition $\lambda=n_{\lambda} \lambda_{0}$ for ${ }^{\forall} \lambda \in \Lambda-\{0\}$ with $\lambda_{0} \in \Lambda_{0}, n_{\lambda} \in \mathbb{Z}$. We also introduce the unique element $\lambda_{1} \in \Lambda_{0}$ by requiring the condition $\left\langle\lambda_{1}, \lambda_{0}\right\rangle=1$. Then, making use of the assumption $\operatorname{gcd}\{N, M\}=1$, we find

$$
\begin{aligned}
\widehat{S}^{(K)}[\lambda] & =\mathbb{Z}_{K} \lambda_{0}+\mathbb{Z}_{n_{\lambda}^{(K)}} \frac{K}{n_{\lambda}^{(K)}} \lambda_{1} \\
& =S^{(K)}[\lambda]+S^{(K)}\left[\lambda+N M \lambda_{1}\right], \quad(K=N, M),
\end{aligned}
$$

where we set

$$
n_{\gamma}^{(K)}:=\operatorname{gcd}\left\{\left|n_{\gamma}\right|, K\right\}, \quad(K=N, M) .
$$

For instance, in the case of $\lambda=m \in \mathbb{Z}-\{0\}$, it is easy to see

$$
\widehat{S}^{(N)}[m]=\mathbb{Z}_{N}+\mathbb{Z}_{n_{m}^{(N)}} \frac{N}{n_{m}^{(N)}} \tau=S^{(N)}[m]+S^{(N)}[m+N M \tau]
$$


and the equality for general case ${ }^{\forall} \lambda \in \Lambda-\{0\}$ follows from modular transformations.

From the above relations, we obtain the following identity,

$$
\left(n_{\lambda}^{(N)} N\right) \mathbf{P}_{\lambda}^{(N)} \mathbf{P}_{\lambda+N M \lambda_{1}}^{(N)}=\sum_{\alpha \in \widehat{S}^{(N)}[\lambda]} \tau_{\alpha}^{(N)},
$$

and a similar relation for $\widetilde{\mathbf{P}}_{*}^{(M)}$. The equality (4.36) implies that $\widehat{Z^{\mathrm{fiber}}}{ }_{\lambda}(\tau)$ given in (4.5) for the even sector $\lambda \in 2 \Lambda-\{0\}$ is identified with the partition function of the type (4.31) with the insertions of $\mathcal{I}_{\lambda}^{(-)}$and $\mathcal{I}_{2\left(\lambda+N M \lambda_{1}\right)}^{(-)}$up to a normalization constant. To be more precise, it is straightforward to show

$$
\begin{aligned}
\widehat{Z^{\text {fiber }}}{ }_{\lambda}(\tau) & =\mathcal{N}_{\lambda}^{\prime} Z^{\text {fiber }}\left[\mathcal{I}_{\lambda}^{(-)}, \mathcal{I}_{2\left(\lambda+N M \lambda_{1}\right)}^{(-)}\right](\tau) \\
& \equiv\left(n_{\lambda}^{(N)} n_{\lambda}^{(M)} N M\right)^{2} Z^{\text {fiber }}\left[\mathcal{I}_{\lambda}^{(-)}, \mathcal{I}_{2\left(\lambda+N M \lambda_{1}\right)}^{(-)}\right](\tau), \quad\left({ }^{\forall} \lambda \in 2 \Lambda-\{0\}\right) .
\end{aligned}
$$

The factor 2 of the subscript in the latter operator assures that the cycle is even, though the actual action is implemented also by $\mathbf{P}_{\lambda+N M \lambda_{1}}^{(N)}$ due to (3.48). Furthermore, the partition function for the odd sector (4.6) is rewritten in the form of (4.32), that is,

$$
\begin{aligned}
\widehat{Z^{\text {fiber }}}{ }_{\lambda}(\tau) & =\mathcal{N}_{\lambda}^{\prime} Z^{\text {fiber }}\left[\mathcal{I}_{\lambda}^{(-)}, \mathcal{I}_{2\left(2 \lambda+N M \lambda_{1}\right)}^{(-)}\right](\tau) \\
& \equiv \mathbf{D}_{[\lambda]} \cdot\left[\mathcal{N}_{2 \lambda}^{\prime} Z^{\text {fiber }}\left[\mathcal{I}_{2 \lambda}^{(-)}, \mathcal{I}_{2\left(2 \lambda+N M \lambda_{1}\right)}^{(-)}\right](\tau)\right] \\
& \equiv\left(n_{2 \lambda}^{(N)} n_{2 \lambda}^{(M)} N M\right) \mathbf{D}_{[\lambda]} \cdot Z^{\text {fiber }}\left[\mathcal{I}_{2 \lambda}^{(-)}, \mathcal{I}_{2\left(2 \lambda+N M \lambda_{1}\right)}^{(-)}\right](\tau), \quad\left({ }^{\forall} \lambda \in \Lambda-2 \Lambda\right) .
\end{aligned}
$$

These are the equalities we have looked for, and show that the fiber partition functions $\widehat{Z^{\text {fiber }}}{ }_{\lambda}(\tau)$ are interpreted to be generated by multiple insertions of the interface operators along different cycles.

\section{Summary and discussion}

In this paper, we studied simple models of non-geometric backgrounds based on the worldsheet CFT equipped with the topological interfaces $[15,17]$. The topological interfaces there composed the twist operators similar to those in orbifold CFTs, and we performed the twisted compactification of the Scherk-Schwarz type [28, 29]. When crossing the defect lines on the world-sheet created by the interface operators, the KK momenta and the winding modes are mixed, which embodies a 'non-geometry' (or the 'stringy geometry', possibly). Requiring the modular invariance, we needed to sum up all the winding sectors with respect to the Scherk-Schwarz circle, or the base space. The fiber CFT, on the other hand, was made up of the world-sheet with the interfaces aligned suitably, so as to correlate in a modular covariant manner to the winding numbers of the base circle.

In our concrete studies, two types of the topological interfaces appeared. One is the group-like defect and the other, which includes the former, is the duality defect $[18,19]$. 
The group-like defect corresponds to the case of $N=M=1$ in section 3.2, and resulted in an example of the T-fold, that is, the Scherk-Schwarz compactification twisted by the T-duality. As we clarified in section 3.5, this model is also T-dualized to the permutation orbifold, which manifestly yields a unitary theory.

On the other hand, the cases of $N M>1$ given in section 3.3 are described in terms of the duality defects that do not implement symmetries in the strict sense. The obtained partition function (3.43) looks rather intricate and the unitarity is not realized as it stands. As mentioned in section 2.3, the topological interfaces in these cases implement a projection that restricts the zero-mode spectrum, instead of an automorphism of the Hilbert space. In order to maintain the unitarity, or conserve the probability, one may thus need to complement this projection by supplying 'twisted sectors' which may differ from those in ordinary orbifold theories. Quite interestingly, a 'unitarization' was indeed possible at least for the cases of $R_{1}=R_{2}=\sqrt{\frac{N}{M}}$, as demonstrated in section 4. Furthermore, the unitarized model is found to be described by the world-sheet with multiple insertions of the interfaces along different cycles. While the even sectors of this model might be identified with some orbifold with the discrete torsion [30,31], the total partition function itself does not seem to correspond to any orbifold conformal theory. ${ }^{8}$

We organized the interface operators, as mentioned in section 3 , so that they act consistently within one Hilbert space. This is in accord with the low-energy analysis that the T-folds lie at the fixed points of the moduli space. Taking also into account our motivation to discuss possible roles of the world-sheet interfaces for string theory, our models may be the simplest from our point of view. Compared with the preceding works [913], where the exact CFT partition functions for T-folds are constructed by identifying some asymmetric twists with particular T-duality transformations, our construction takes a different route based on the interfaces. In the case of the group-like defect, our resultant model, however, fits into the same category of asymmetric orbifolds, except that our model has a continuous modulus in a fixed line instead of isolated fixed points. In the case of the duality defects, our construction would be a novel type other than ordinary asymmetric orbifolds. In both cases, our approach would provide a unified picture for the non-geometric backgrounds of T-fold type. Our approach may also be generalized to a large class of models, as long as the action of the interfaces is well-defined within one Hilbert space, which implies that the model is on the fixed submanifold in the moduli space.

The analysis of non-geometric backgrounds typically involves compactification radii of or below the string scale. The advantage of the world-sheet CFT approach is that the results are $\alpha^{\prime}$-exact and thus valid even at the string scale, where the notion of classical geometry may not be valid. When the string coupling for the genus expansion becomes large, the world-sheet approach here is not applicable, and should be superseded by nonperturbative approaches.

\footnotetext{
${ }^{8}$ It would be worth mentioning that, in the papers [34-36], the authors have been investigating the 'generalized orbifolds' that do not arise from any symmetry group, for example, in the context of the topological Landau-Ginzburg theories with defects.
} 
For future work, if intending the applications to string compactifications, we would have several tasks to be done:

- the construction of the unitarized models for general radii $R_{1}, R_{2}$ satisfying the condition (3.2);

- the extension to the case of the fiber CFT on higher dimensional tori;

- the supersymmetric extension by means of superconformal interfaces;

- the target-space interpretation of the insertions of the interfaces and the resultant models.

Especially, the topological interfaces in $\mathcal{N}=1$ SCFTs on higher dimensional tori have been elaborated in [26]. It has been clarified there that the duality (or the 'orbifold equivalence') defects which do not preserve the charge lattice of string theory generically correspond to the $O(d, d ; \mathbb{Q})$-group for $d$-dimensional torus. It is an interesting question whether one can construct the unitary and modular invariant models based on such $O(d, d ; \mathbb{Q})$ interfaces as extensions of the unitarized model proposed in this paper. It would also be interesting to see, as mentioned in [26], that an object representing the 'quasi-symmetry' survives even under the $\alpha^{\prime}$ corrections and plays a definite role in string theory.

Moreover, a natural direction of the future studies would be the extensions to nontrivial cases with $\mathcal{N}=2 \mathrm{SCFTs}$, say, the Gepner models as the fiber CFT, in which one would handle the duality defects inducing the mirror transformations. Of course, one may imagine the models of Scherk-Schwarz compactifications with the self-dual mirror twisting acting on some $\mathcal{N}=4$ fiber SCFTs, similarly to [37]. These cases may be described by the group-like defects and reduce to asymmetric orbifolds. However, more general duality defects made up of the $\mathcal{N}=2$ topological interfaces would be capable of producing a much broader class of superstring vacua, which would be fairly non-trivial and curious.

Another interesting direction to be pursued would be the possibility of more general unitary models by multiple insertions of the interface operators. In section 4.3, we chose particular cycles along which the interfaces are aligned, in order to obtain the unitarized model (4.8). However, it is not yet clear what the general principle is, in order for the configuration of the interfaces to realize sensible unitary models. In any case, we hope that this work would serve as a step to construct a novel type of string vacua based on the world-sheets equipped with the topological interfaces or the interface operators composed of them.

\section{Acknowledgments}

We would like to thank K. Sakai for useful comments on the conformal interfaces. This work is supported in part by JSPS KAKENHI Grant Number 24540248 and 23540322 from Japan Society for the Promotion of Science (JSPS). 


\section{A Conventions for theta functions and some useful formulas}

\section{Theta functions:}

$$
\begin{aligned}
\theta_{1}(\tau, z) & =i \sum_{n=-\infty}^{\infty}(-1)^{n} q^{(n-1 / 2)^{2} / 2} y^{n-1 / 2} \\
& \equiv 2 \sin (\pi z) q^{1 / 8} \prod_{m=1}^{\infty}\left(1-q^{m}\right)\left(1-y q^{m}\right)\left(1-y^{-1} q^{m}\right) \\
\theta_{2}(\tau, z) & =\sum_{n=-\infty}^{\infty} q^{(n-1 / 2)^{2} / 2} y^{n-1 / 2} \equiv 2 \cos (\pi z) q^{1 / 8} \prod_{m=1}^{\infty}\left(1-q^{m}\right)\left(1+y q^{m}\right)\left(1+y^{-1} q^{m}\right) \\
\theta_{3}(\tau, z) & =\sum_{n=-\infty}^{\infty} q^{n^{2} / 2} y^{n} \equiv \prod_{m=1}^{\infty}\left(1-q^{m}\right)\left(1+y q^{m-1 / 2}\right)\left(1+y^{-1} q^{m-1 / 2}\right), \\
\theta_{4}(\tau, z) & =\sum_{n=-\infty}^{\infty}(-1)^{n} q^{n^{2} / 2} y^{n} \equiv \prod_{m=1}^{\infty}\left(1-q^{m}\right)\left(1-y q^{m-1 / 2}\right)\left(1-y^{-1} q^{m-1 / 2}\right) . \\
\Theta_{m, k}(\tau, z) & =\sum_{n=-\infty}^{\infty} q^{k\left(n+\frac{m}{2 k}\right)^{2}} y^{k\left(n+\frac{m}{2 k}\right)} \\
\eta(\tau) & =q^{1 / 24} \prod_{n=1}^{\infty}\left(1-q^{n}\right) .
\end{aligned}
$$

Here, we have set $q:=e^{2 \pi i \tau}, y:=e^{2 \pi i z}$.

We use abbreviations, $\theta_{i}(\tau) \equiv \theta_{i}(\tau, 0)\left(\theta_{1}(\tau) \equiv 0\right), \Theta_{m, k}(\tau) \equiv \Theta_{m, k}(\tau, 0)$. We also set

$$
Z^{(k)}[u, v](\tau):=\frac{1}{|\eta(\tau)|^{2}} \Theta_{u+v, k}(\tau) \overline{\Theta_{-u+v, k}(\tau)},
$$

which is often used in the main text.

\section{Product formula of theta function:}

$$
\Theta_{m, k}(\tau, z) \Theta_{m^{\prime}, k^{\prime}}\left(\tau, z^{\prime}\right)=\sum_{r \in \mathbb{Z}_{k+k^{\prime}}} \Theta_{m k^{\prime}-m^{\prime} k+2 k k^{\prime} r, k k^{\prime}\left(k+k^{\prime}\right)}(\tau, u) \Theta_{m+m^{\prime}+2 k r, k+k^{\prime}}(\tau, v),
$$

where we set $u=\frac{z-z^{\prime}}{k+k^{\prime}}, v=\frac{k z+k^{\prime} z^{\prime}}{k+k^{\prime}}$.

\section{Poisson resummation formula:}

$$
\begin{array}{r}
\sum_{n \in \mathbb{Z}} \exp \left(-\pi \alpha(n+a)^{2}+2 \pi i b(n+a)\right)=\frac{1}{\sqrt{\alpha}} \sum_{m \in \mathbb{Z}} \exp \left(-\frac{\pi(m-b)^{2}}{\alpha}+2 \pi i m a\right), \\
(\alpha>0, a, b \in \mathbb{R}) .
\end{array}
$$




\section{Modular invariant 'inner product':}

$$
\langle\alpha, \beta\rangle_{\tau}:=\frac{1}{\tau_{2}} \operatorname{Im}(\alpha \bar{\beta}) .
$$

For example, when $\alpha \equiv x_{1} \tau+x_{2}, \beta \equiv y_{1} \tau+y_{2}$, one obtains $\langle\alpha, \beta\rangle_{\tau}=x_{1} y_{2}-x_{2} y_{1}$. The inner product $\langle,\rangle_{\tau}$ is modular invariant, that is,

$$
\langle\alpha, \beta\rangle_{\tau+1}=\langle\alpha, \beta\rangle_{\tau}, \quad\left\langle\frac{\alpha}{\tau}, \frac{\beta}{\tau}\right\rangle_{-\frac{1}{\tau}}=\langle\alpha, \beta\rangle_{\tau} .
$$

We also note

$$
\langle\alpha, \beta\rangle_{\ell \tau}=\frac{1}{\ell}\langle\alpha, \beta\rangle_{\tau}, \quad\left({ }^{\forall} \ell \neq 0\right) .
$$

We often use the abbreviation $\langle,\rangle \equiv\langle,\rangle_{\tau}$ in the cases with no fear of confusion.

\section{B Diagonal part operator}

In this appendix we present the definition of the 'diagonal part operator' $\mathbf{D}_{[\lambda]}([\lambda] \in \Lambda / 2 \Lambda$, ${ }^{\forall} \lambda \in \Lambda-2 \Lambda$ ) repeatedly used in the main text.

As a preliminary, we start with a general function $F_{(w, m)}(\tau)(w, m \in \mathbb{Z})$ of the form such as

$$
F_{(w, m)}(\tau)=\sum_{\ell, \tilde{\ell}} \sum_{n, \tilde{n} \in \mathbb{Z}} c(\ell, \tilde{\ell}, n, \tilde{n} ; w, m) q^{\Delta(\ell)+n} \overline{q^{\tilde{\Delta}(\tilde{\ell})+\tilde{n}}}
$$

that possesses the following properties,

$$
F_{(-w,-m)}(\tau)=F_{(w, m)}(\tau), \quad F_{(w, m)}(\tau+1)=F_{(w, w+m)}(\tau), \quad F_{(w, m)}\left(-\frac{1}{\tau}\right)=F_{(m,-w)}(\tau) .
$$

We identify the 'partition function' whose diagonal part is of our interest as

$$
\mathbf{Z}_{(w, m)}(\tau) \equiv F_{(w, m)}(\tau)^{2}
$$

and regard the two factors $F_{(w, m)}(\tau)$ as the contributions from $\mathrm{CFT}_{1}$ and $\mathrm{CFT}_{2} \cdot{ }^{9}$

Then, we define the 'diagonal part operator' $\mathbf{D}_{[\lambda]}\left({ }^{\forall} \lambda \in \Lambda-2 \Lambda,[\lambda] \in \Lambda / 2 \Lambda\right.$ denotes the representative of $\lambda$ ) by

$$
\mathbf{D}_{[w \tau+m]} \cdot \mathbf{Z}_{(w, m)}(\tau):= \begin{cases}F_{\left(\frac{w}{2}, m\right)}(2 \tau), & (w \in 2 \mathbb{Z}, m \in 2 \mathbb{Z}+1), \\ F_{\left(w, \frac{m}{2}\right)}\left(\frac{\tau}{2}\right), & (w \in 2 \mathbb{Z}+1, m \in 2 \mathbb{Z}), \\ F_{\left(w, \frac{m-w}{2}\right)}\left(\frac{\tau+1}{2}\right), & (w \in 2 \mathbb{Z}+1, m \in 2 \mathbb{Z}+1) .\end{cases}
$$

\footnotetext{
${ }^{9}$ Of course, a natural extension of the argument given here would be the diagonal part operator acting on a more general partition function such as $\mathbf{Z}_{(w, m)}(\tau) \equiv F_{(w, m)}^{(1)}(\tau) F_{(w, m)}^{(2)}(\tau), F_{(w, m)}^{(1)}(\tau) \neq F_{(w, m)}^{(2)}(\tau)$. In this paper, however, it is sufficient to restrict to the simple case (B.3).
} 
Alternatively, if adopting the notation such as $F_{w \tau+m}(\tau) \equiv F_{(w, m)}(\tau), \mathbf{Z}_{w \tau+m}(\tau) \equiv$ $\mathbf{Z}_{(w, m)}(\tau)$, we can rewrite it as

$$
\mathbf{D}_{[w \tau+m]} \cdot \mathbf{Z}_{w \tau+m}(\tau):= \begin{cases}F_{\frac{w}{2} \cdot 2 \tau+m}(2 \tau) \equiv F_{w \tau+m}(2 \tau), & (w \in 2 \mathbb{Z}, m \in 2 \mathbb{Z}+1), \\ F_{w \frac{\tau}{2}+\frac{m}{2}}\left(\frac{\tau}{2}\right) \equiv F_{\frac{1}{2}(w \tau+m)}\left(\frac{\tau}{2}\right), & (w \in 2 \mathbb{Z}+1, m \in 2 \mathbb{Z}), \\ F_{w \frac{\tau+1}{2}+\frac{m-w}{2}}\left(\frac{\tau+1}{2}\right) \equiv F_{\frac{1}{2}(w \tau+m)}\left(\frac{\tau+1}{2}\right) . & (w \in 2 \mathbb{Z}+1, m \in 2 \mathbb{Z}+1) .\end{cases}
$$

For ${ }^{\forall} \lambda=m \in 2 \mathbb{Z}+1, \mathbf{D}_{[m]} \cdot \mathbf{Z}_{m}(\tau)$ is literally interpreted as the diagonal part of the partition function $\mathbf{Z}_{m}(\tau)$, which means

$$
\mathbf{D}_{[m]} \cdot \mathbf{Z}_{m}(\tau)=F_{(0, m)}(2 \tau) \equiv \sum_{\ell, \tilde{\ell}} \sum_{n, \tilde{n} \in \mathbb{Z}} c(\ell, \tilde{\ell}, n, \tilde{n} ; 0, m) q^{2(\Delta(\ell)+n)} \overline{q^{2(\widetilde{\Delta}(\tilde{\ell})+\tilde{n})}} .
$$

Furthermore, the function $\mathbf{D}_{[\lambda]} \cdot \mathbf{Z}_{\lambda}(\tau)$ possesses the expected modularity; namely, the identities,

$$
\mathbf{D}_{[\lambda]} \cdot \mathbf{Z}_{\lambda}(\tau+1)=\mathbf{D}_{[\lambda]} \cdot \mathbf{Z}_{\lambda}(\tau), \quad \mathbf{D}_{[\lambda / \tau]} \cdot \mathbf{Z}_{\lambda / \tau}\left(-\frac{1}{\tau}\right)=\mathbf{D}_{[\lambda]} \cdot \mathbf{Z}_{\lambda}(\tau)
$$

are satisfied. In fact, almost all of the identities are obvious from the definition (B.4), and it is only non-trivial to prove

$$
\mathbf{D}_{[\lambda / \tau]} \cdot \mathbf{Z}_{\lambda / \tau}\left(-\frac{1}{\tau}\right)=\mathbf{D}_{[\lambda]} \cdot \mathbf{Z}_{\lambda}(\tau), \quad\left({ }^{\forall} \lambda \in(2 \mathbb{Z}+1) \tau+(2 \mathbb{Z}+1)\right) .
$$

This is equivalent to the identity,

$$
F_{\left(w, \frac{m-w}{2}\right)}\left(\frac{-\frac{1}{\tau}+1}{2}\right)=F_{\left(m,-\frac{w+m}{2}\right)}\left(\frac{\tau+1}{2}\right), \quad\left({ }^{\forall} w, m \in 2 \mathbb{Z}+1\right),
$$

which follows from (B.2) as is easily checked.

Open Access. This article is distributed under the terms of the Creative Commons Attribution License (CC-BY 4.0), which permits any use, distribution and reproduction in any medium, provided the original author(s) and source are credited.

\section{References}

[1] A. Dabholkar and C. Hull, Duality twists, orbifolds and fluxes, JHEP 09 (2003) 054 [hep-th/0210209] [INSPIRE].

[2] S. Hellerman, J. McGreevy and B. Williams, Geometric constructions of nongeometric string theories, JHEP 01 (2004) 024 [hep-th/0208174] [INSPIRE].

[3] A. Flournoy, B. Wecht and B. Williams, Constructing nongeometric vacua in string theory, Nucl. Phys. B 706 (2005) 127 [hep-th/0404217] [INSPIRE].

[4] C.M. Hull, A Geometry for non-geometric string backgrounds, JHEP 10 (2005) 065 [hep-th/0406102] [INSPIRE]. 
[5] J. Shelton, W. Taylor and B. Wecht, Nongeometric flux compactifications, JHEP 10 (2005) 085 [hep-th/0508133] [INSPIRE].

[6] J. Shelton, W. Taylor and B. Wecht, Generalized Flux Vacua, JHEP 02 (2007) 095 [hep-th/0607015] [INSPIRE].

[7] A. Dabholkar and C. Hull, Generalised T-duality and non-geometric backgrounds, JHEP 05 (2006) 009 [hep-th/0512005] [INSPIRE].

[8] K.S. Narain, M.H. Sarmadi and C. Vafa, Asymmetric Orbifolds, Nucl. Phys. B 288 (1987) 551 [INSPIRE].

[9] A. Flournoy and B. Williams, Nongeometry, duality twists and the worldsheet, JHEP 01 (2006) 166 [hep-th/0511126] [INSPIRE].

[10] S. Hellerman and J. Walcher, Worldsheet CFTs for Flat Monodrofolds, hep-th/0604191 [INSPIRE].

[11] S. Kawai and Y. Sugawara, D-branes in T-fold conformal field theory, JHEP 02 (2008) 027 [arXiv: 0709.0257] [INSPIRE].

[12] C. Condeescu, I. Florakis and D. Lüst, Asymmetric Orbifolds, Non-Geometric Fluxes and Non-Commutativity in Closed String Theory, JHEP 04 (2012) 121 [arXiv:1202.6366] [INSPIRE].

[13] C. Condeescu, I. Florakis, C. Kounnas and D. Lüst, Gauged supergravities and non-geometric Q/R-fluxes from asymmetric orbifold CFT's, JHEP 10 (2013) 057 [arXiv:1307.0999] [INSPIRE].

[14] E. Wong and I. Affleck, Tunneling in quantum wires: A Boundary conformal field theory approach, Nucl. Phys. B 417 (1994) 403 [INSPIRE].

[15] V.B. Petkova and J.B. Zuber, Generalized twisted partition functions, Phys. Lett. B 504 (2001) 157 [hep-th/0011021] [INSPIRE].

[16] C. Bachas, J. de Boer, R. Dijkgraaf and H. Ooguri, Permeable conformal walls and holography, JHEP 06 (2002) 027 [hep-th/0111210] [INSPIRE].

[17] C. Bachas and M. Gaberdiel, Loop operators and the Kondo problem, JHEP 11 (2004) 065 [hep-th/0411067] [INSPIRE].

[18] J. Fröhlich, J. Fuchs, I. Runkel and C. Schweigert, Kramers-Wannier duality from conformal defects, Phys. Rev. Lett. 93 (2004) 070601 [cond-mat/0404051] [INSPIRE].

[19] J. Fröhlich, J. Fuchs, I. Runkel and C. Schweigert, Duality and defects in rational conformal field theory, Nucl. Phys. B 763 (2007) 354 [hep-th/0607247] [INSPIRE].

[20] J. Fuchs, C. Schweigert and K. Waldorf, Bi-branes: target space geometry for world sheet topological defects, J. Geom. Phys. 58 (2008) 576 [hep-th/0703145] [INSPIRE].

[21] K. Graham and G.M.T. Watts, Defect lines and boundary flows, JHEP 04 (2004) 019 [hep-th/0306167] [INSPIRE].

[22] I. Brunner and D. Roggenkamp, Defects and bulk perturbations of boundary Landau-Ginzburg orbifolds, JHEP 04 (2008) 001 [arXiv:0712.0188] [INSPIRE].

[23] D. Gaiotto, Domain walls for two-dimensional renormalization group flows, JHEP 12 (2012) 103 [arXiv: 1201.0767] [INSPIRE]. 
[24] C. Bachas and I. Brunner, Fusion of conformal interfaces, JHEP 02 (2008) 085 [arXiv:0712.0076] [INSPIRE].

[25] Y. Satoh, On supersymmetric interfaces for string theory, JHEP 03 (2012) 072 [arXiv:1112.5935] [INSPIRE].

[26] C. Bachas, I. Brunner and D. Roggenkamp, A worldsheet extension of $O(d, d: Z)$, JHEP 10 (2012) 039 [arXiv: 1205.4647] [INSPIRE].

[27] S. Elitzur, B. Karni, E. Rabinovici and G. Sarkissian, Defects, Super-Poincaré line bundle and Fermionic T-duality, JHEP 04 (2013) 088 [arXiv: 1301.6639] [INSPIRE].

[28] J. Scherk and J.H. Schwarz, Spontaneous breaking of supersymmetry through dimensional reduction, Phys. Lett. B 82 (1979) 60 [InSPIRE].

[29] J. Scherk and J.H. Schwarz, How to get masses from extra dimensions, Nucl. Phys. B 153 (1979) 61 [inSPIRE].

[30] C. Vafa, Modular invariance and discrete torsion on orbifolds, Nucl. Phys. B 273 (1986) 592 [INSPIRE].

[31] C. Vafa and E. Witten, On orbifolds with discrete torsion, J. Geom. Phys. 15 (1995) 189 [hep-th/9409188] [INSPIRE].

[32] J. Fuchs, M.R. Gaberdiel, I. Runkel and C. Schweigert, Topological defects for the free boson CFT, J. Phys. A 40 (2007) 11403 [arXiv:0705.3129] [inSPIRE].

[33] K. Sakai and Y. Satoh, Entanglement through conformal interfaces, JHEP 12 (2008) 001 [arXiv:0809.4548] [INSPIRE].

[34] J. Fröhlich, J. Fuchs, I. Runkel and C. Schweigert, Defect lines, dualities and generalised orbifolds, arXiv:0909.5013 [INSPIRE].

[35] N. Carqueville and I. Runkel, Orbifold completion of defect bicategories, arXiv:1210.6363 [INSPIRE].

[36] I. Brunner, N. Carqueville and D. Plencner, Orbifolds and topological defects, Commun. Math. Phys. 332 (2014) 669 [arXiv:1307.3141] [INSPIRE].

[37] S. Kawai and Y. Sugawara, Mirrorfolds with K3 fibrations, JHEP 02 (2008) 065 [arXiv:0711.1045] [INSPIRE]. 